\title{
A class of continuous non-associative algebras arising from algebraic groups including $E_{8}$
}

\author{
Maurice Chayet ${ }^{1}$ and Skip Garibaldi ${ }^{D_{2}}$ \\ ${ }^{1}$ ECAM-EPMI, 13 Boulevard de l'Hautil, 95092 Cergy Pointoise Cedex, France; E-mail: m.chayet@ecam-epmi.com. \\ ${ }^{2}$ IDA Center for Communications Research-La Jolla, 4320 Westerra Ct, San Diego, CA 92121, USA; \\ E-mail: skip@garibaldibros.com.
}

Received: 18 June 2020; Revised: 12 November 2020; Accepted: 3 December 2020

2020 Mathematics Subject Classification: Primary - 17B25; Secondary - 17D99, 20G41

\begin{abstract}
We give a construction that takes a simple linear algebraic group $G$ over a field and produces a commutative, unital, and simple non-associative algebra $A$ over that field. Two attractions of this construction are that (1) when $G$ has type $E_{8}$, the algebra $A$ is obtained by adjoining a unit to the 3875-dimensional representation; and (2) it is effective, in that the product operation on $A$ can be implemented on a computer. A description of the algebra in the $E_{8}$ case has been requested for some time, and interest has been increased by the recent proof that $E_{8}$ is the full automorphism group of that algebra. The algebras obtained by our construction have an unusual Peirce spectrum.
\end{abstract}

\section{Introduction}

We present a construction that takes an absolutely simple linear algebraic group $G$ over a field $k$ and produces a commutative, unital non-associative algebra that we denote by $A(\mathfrak{g})$. As a vector space, $A(\mathfrak{g})$ is a subspace of the symmetric square $S^{2} \mathfrak{g}$ of the Lie algebra $\mathfrak{g}$ of $G$. We give an explicit formula (4.1) for the product on $A(\mathfrak{g})$, which makes our construction effective in the sense that one can perform computer calculations (Section 11), although we do not rely on computer calculations for our results. There is a natural symmetric bilinear form on $A(\mathfrak{g})$, which we show is associative (Section 6 ) and nondegenerate (Section 8) and positive-definite in case $k=\mathbb{R}$ and $G$ is compact. We leverage this and the structure of $A(\mathfrak{g})$ as a representation of $G$ to show that it is a simple $k$-algebra (Corollary 8.6).

This work may be viewed in the context of the general problem of describing exceptional groups as automorphism groups, which dates back to Killing's 1889 paper [25]. As an example, the Lie group $G_{2}$ can be viewed as the automorphism group of the octonions (E. Cartan [9]), the stabilizer of a cross product on $\mathbb{R}^{7}$ (F. Engel, [16], [20]), or the symmetry group for a ball of radius 1 rolling on a fixed ball of radius 3 without slipping or twisting (E. Cartan, [3]). For $E_{8}$, it is known from [19] that it is the identity component of the stabilizer of an octic form on the Lie algebra $e_{8}$ and that it is the automorphism group of the $E_{8}$-invariant algebra on its 3875-dimensional irreducible representation. (See also [18, Section 3] or [19, Section 16] for broader discussions of other realizations.) The latter description of $E_{8}$ is known to be true even though this algebra is not well-understood; this paper gives explicit and effective formulas for calculating in the algebra. We note here that $\operatorname{Aut}\left(A\left(\mathfrak{e}_{8}\right)\right)=E_{8}$; see Proposition 9.1.

The algebras $A(\mathfrak{g})$ constructed here are 'non-generic' in the sense of [28], meaning that $A(\mathfrak{g}) \otimes \bar{k}$ contains infinitely many idempotents, for $\bar{k}$ an algebraic closure of $k$. Moreover, the Peirce spectrum

(C) The Author(s), 2021. Published by Cambridge University Press. This is an Open Access article, distributed under the terms of the Creative Commons Attribution licence (http://creativecommons.org/licenses/by/4.0/), which permits unrestricted re-use, distribution, and reproduction in any medium, provided the original work is properly cited. 
of $A(\mathfrak{g}) \otimes \bar{k}$, that is, the union of the set of eigenvalues for left multiplication by $u$ as $u$ varies over idempotents of $A(\mathfrak{g}) \otimes \bar{k}$, is infinite; see Example 4.9. In case $k=\mathbb{R}$ and apart from types $A_{1}$ and $A_{2}$, this collection of eigenvalues includes the unit interval, and consequently one might call these algebras 'continuous' as we have done in the title of the article. We remark that this kind of situation - where a popular property holds for generic cases but fails for a structure naturally associated with a simple algebraic group $G-$ is familiar from the study of homogeneous $G$-invariant polynomials. In that setting, a generic homogeneous polynomial is non-singular, yet $G$-invariant polynomials of degree $\geq 3$ are singular [35], such as the determinant on $n$-by- $n$ matrices.

Ignoring some very small cases, the algebras $A(\mathfrak{g})$ are not power-associative. This is not a defect of our construction. We show that even if one alters the one choice we made in the construction, the resulting algebra would still not be power-associative; see Proposition 5.3(2) and Remark 9.2.

In the penultimate section, Section 10, we give an alternative realization of $A(\mathfrak{g})$ inside End $(V)$, where $V$ is the natural module for $G$ of type $A_{2}, G_{2}, F_{4}, E_{6}$, or $E_{7}$. We use this alternative realization to explicitly compute $A\left(\mathfrak{s l}_{3}\right)$ (Example 10.9). We conclude with an appendix (Appendix A) giving various results about adding a unit to a non-associative algebra that we refer to in the body of the paper.

We work over a rather general field $k$ and do not assume that $G$ is split, although our results are new already in the case where $k$ is the complex numbers $\mathbb{C}$. The additional generality comes at hardly any cost due to the tools we use. Readers who are not interested in the full generality are invited to assume throughout that $k=\mathbb{C}$ and identify the symbols $H^{0}(\lambda)=V(\lambda)=L(\lambda)$.

An unusual feature of our work is that the case where $G$ is of type $E_{8}$ is less complicated than other $G$ in several ways, at least when $k=\mathbb{C}$. For $E_{8}$, one has extra formulas to use, such as Okubo's Identity $\operatorname{Tr}\left(\pi(X)^{4}\right)=\alpha_{\pi} K(X, X)^{4}$ (Lemma 10.1, which holds for all $G$ of exceptional type) and a similar identity for $\operatorname{Tr}\left(\pi(X)^{6}\right)$ (which holds for type $E_{8}$ ). Another way that $E_{8}$ is less complicated is that the Molien series $1+t^{2}+t^{3}+3 t^{4}+3 t^{5}+10 t^{6}+16 t^{7}+\cdots$ for $E_{8}$ acting on its 3875-dimensional representation $V$ has coefficients no greater than the Molien series for the corresponding representations of other groups of type $E, F$ or $G$. Yet another way is that the second symmetric power $\mathrm{S}^{2} V$ is a sum of 6 irreducible terms, which is minimal among the types $E, F$ and $G$.

Our original approach to the material in this paper was to focus on the case of $E_{8}$ and leverage these tools. In this way, we discovered the product formula on $A(\mathfrak{g})$, and only in hindsight did we see that it was a general construction that worked for all simple $G$. Due to this inverted approach, preparing this document took more than three years. Just before we intended to release this work on arXiv, the paper [11] appeared, which studies algebras that are almost the same, albeit restricted to the cases where the root system of $G$ is simply laced and $G$ is split and char $k=0$; see Remark 4.6 below. Both that article and this one view the algebras as subspaces of $S^{2} \mathfrak{g}$ and provide an associative symmetric bilinear form (we say $A(\mathfrak{g})$ is metrized, whereas they say Frobenius), but from there our approaches and results diverge.

\section{Background material}

Let $k$ be a field of characteristic different from 2, and suppose that $\mathfrak{g}$ is a Lie algebra over $k$ whose Killing form, $K$, is nondegenerate. Then the $k$-algebra of linear transformations of $\mathfrak{g}$, denoted $\operatorname{End}(\mathfrak{g})$, has a 'transpose' operator $\mathrm{T}$ given by

$$
K(T(X), Y)=K\left(X, T^{\top}(Y)\right) \quad \text { for } T \in \operatorname{End}(\mathfrak{g}) \text { and } X, Y \in \mathfrak{g} .
$$

\section{Identification of representations}

Another way to view the nondegeneracy of $K$ is that it provides a $\mathfrak{g}$-equivariant isomorphism of $\mathfrak{g}$-representations

$$
\mathfrak{g} \stackrel{\sim}{\rightarrow} \mathfrak{g}^{*} \quad \text { via } \quad X \mapsto K(X,-) .
$$


This identification extends to an isomorphism of $\mathfrak{g}$-modules

$$
\mathfrak{g} \otimes \mathfrak{g} \stackrel{\sim}{\rightarrow} \mathfrak{g} \otimes \mathfrak{g}^{*}=\operatorname{End}(\mathfrak{g}) .
$$

As char $k \neq 2$, the natural surjection of $\mathfrak{g} \otimes \mathfrak{g}$ onto the second symmetric power $S^{2} \mathfrak{g}$ is split by the map

$$
\mathrm{S}^{2} \mathfrak{g} \hookrightarrow \mathfrak{g} \otimes \mathfrak{g} \quad \text { given by } X Y \mapsto \frac{1}{2}(X \otimes Y+Y \otimes X) .
$$

Definition 2.4. Define $P: S^{2} \mathfrak{g} \hookrightarrow \operatorname{End}(\mathfrak{g})$ as the composition of (2.2) with (2.3). It is $\mathfrak{g}$-equivariant, and its image is the space

$$
\mathcal{H}(\mathfrak{g}):=\left\{T \in \operatorname{End}(\mathfrak{g}) \mid T^{\top}=T\right\}
$$

of symmetric operators. We have:

$$
P(X Y)=\frac{1}{2}[X \otimes K(Y,-)+Y \otimes K(X,-)] \quad \text { for } X, Y \in \mathfrak{g} .
$$

Example 2.5. For $\left\{X_{i}\right\}$ a basis of $\mathfrak{g}$ and $\left\{Y_{i}\right\}$ the dual basis with respect to $K$, set $e_{\otimes}:=\sum X_{i} \otimes Y_{i} \in \mathfrak{g} \otimes \mathfrak{g}$ and $e_{\mathrm{S}}:=\sum X_{i} Y_{i}$, the image of $e_{\otimes}$ in $\mathrm{S}^{2} \mathfrak{g}$. Neither $e_{\otimes}$ nor $e_{\mathrm{S}}$ depends on the choice of the $X_{i} \mathrm{~s}$. Moreover, the identification (2.2) sends $e_{\otimes} \mapsto \mathrm{Id}_{\mathfrak{g}}$, so $P\left(e_{\mathrm{S}}\right)=\mathrm{Id}_{\mathfrak{g}}$.

The spaces End $(\mathfrak{g})$ and $\mathcal{H}(\mathfrak{g})$ are Jordan algebras under the Jordan product $\bullet$ defined by

$$
T \bullet U:=\frac{1}{2}(T U+U T) \quad \text { for } T, U \in \operatorname{End}(\mathfrak{g}) .
$$

Example 2.7. For $X_{1}, X_{2}, X_{3}, X_{4} \in \mathfrak{g}$, we have:

$$
\begin{aligned}
P\left(X_{1} X_{2}\right) \bullet P\left(X_{3} X_{4}\right)=\frac{1}{4}\left[K\left(X_{1}, X_{3}\right) P\left(X_{2} X_{4}\right)+K\left(X_{1}, X_{4}\right) P\left(X_{2} X_{3}\right)\right. & \\
& \left.+K\left(X_{2}, X_{3}\right) P\left(X_{1} X_{4}\right)+K\left(X_{2}, X_{4}\right) P\left(X_{1} X_{3}\right)\right] .
\end{aligned}
$$

Therefore, for any subspace $\mathfrak{I}$ of $\mathfrak{g}, P\left(S^{2} \mathfrak{l}\right)$ is a Jordan subalgebra of $\mathcal{H}(\mathfrak{g})$. If $K(X, X) \neq 0$, then the element $P\left(X^{2}\right) / K(X, X)$ is an idempotent in the Jordan algebra.

Suppose that furthermore $\mathfrak{I}$ has an orthonormal basis $X_{1}, \ldots, X_{r}$. Then for $i \neq j, P\left(X_{i}^{2}\right) \bullet P\left(X_{j}^{2}\right)=0$ and $P\left(X_{i}^{2}\right) \bullet P\left(X_{i} X_{j}\right)=\frac{1}{2} P\left(X_{i} X_{j}\right)$. In particular, $\sum P\left(X_{i}^{2}\right)$ is the identity element in $P\left(\mathrm{~S}^{2} \mathfrak{l}\right)$.

\section{Global hypotheses}

We now add hypotheses that will be assumed until the start of Appendix A. We will assume that $\mathfrak{g}$ is the Lie algebra of an absolutely simple linear algebraic group $G$ over $k$. That is, $G$ is a smooth affine group scheme of finite type over $k$, and $G \times \bar{k}$ is simple: $G \times \bar{k}$ is connected, semisimple (= has trivial radical) and $\neq 1$, and its associated root system is irreducible.

We write $h$ for the Coxeter number and $h^{\vee}$ for the dual Coxeter number of (the root system of) $G$; some examples are given in Table 1 below. It is true that $\operatorname{rank} G<h^{\vee} \leq h$, and the root system of $G$ is simply laced if and only if $h^{\vee}=h$.

We additionally assume until the start of the appendix that char $k$ is zero or at least $h+2$. Consequently, the integers $2, \operatorname{rank} G, h^{\vee}, h^{\vee}+1$ are not zero in $k$, so the same is true for $\operatorname{dim} G=(\operatorname{rank} G)(h+1)$. Examining the type of $G$ in turn, we find: (1) the characteristic is 'very good' for $G$; (2) the determinant of the Cartan matrix is not zero in $k$; and (3) the ratio $v_{G}$ of the square length of a long root to that of a short root (equivalently, the valence of the Dynkin diagram of $G$ ) is not zero in $k$.

The discriminant of the Killing form $K$ on $\mathfrak{g}$ can be expressed as a product of integers we have already observed are not zero in $k$ [43, p. E-14, I.4.8(a)], and therefore $K$ is nondegenerate. Finally, $\mathfrak{g}$ is 
a simple Lie algebra that is an irreducible representation of $G$ [21]; it follows that, if $G^{\prime}$ is isogenous to $G$, then $\mathfrak{g}^{\prime} \cong \mathfrak{g}$.

\section{Representations}

Suppose $G$ is split, and put $\mathfrak{h}$ for the Lie algebra of a split maximal torus $T$. For a dominant weight $\lambda \in T^{*}$, we write $L(\lambda)$ for the irreducible representation of $G$ with highest weight $\lambda$. The dimension and character of $L(\lambda)$ may depend on the characteristic of $k$ and not just on root system data. However, there are representations $H^{0}(\lambda)$ and $V(\lambda)$ of $G$, both with highest weight $\lambda$, which equal $L(\lambda)$ when char $k$ is zero or 'big enough' (where what counts as big enough depends on $G$ and $\lambda$ ), and whose character is the same as the character of the irreducible representation over $\mathbb{C}$ with highest weight $\lambda$. The representations $V(\lambda)$ are called Weyl modules; a basic example of such is the tautological representation of $\mathrm{SO}_{n}$. See [23] for background on these representations. We use the fact that these representations are defined over $\mathbb{Z}$; see [23, II.8.3]. See also Section 7 for a discussion of the case where $G$ is not assumed to be split.

\section{Casimir operator}

Put $\langle\mid\rangle$ for the canonical bilinear form on the weight lattice of $G$, as defined in [6, Section VI.1.12] or [15, p. 115]; it is the unique nonzero and Weyl-group-invariant inner product satisfying $\left\langle\lambda \mid \lambda^{\prime}\right\rangle=$ $\sum_{\alpha}\langle\lambda \mid \alpha\rangle\left\langle\lambda^{\prime} \mid \alpha\right\rangle$, where $\alpha$ varies over the roots. Then $\langle\alpha \mid \alpha\rangle=1 / h^{\vee}$ for every long root $\alpha$; see [45, p. 150].

More generally, for each root $\alpha$, define $v_{\alpha}:=1$ if $\alpha$ is long and $v_{\alpha}:=v_{G}$ if $\alpha$ is short. By definition, then, $\langle\alpha \mid \alpha\rangle=\left(v_{\alpha} h^{\vee}\right)^{-1}$ for every root $\alpha$, and this is not zero in $k$.

For the next two lemmas, we set $R:=\mathbb{Z}_{(\operatorname{char} k)}$, the subring of $\mathbb{Q}$ whose nonzero elements are the fractions with denominator not divisible by char $k$. Note that $R$ is a local $\operatorname{ring}, R /($ char $k) \subseteq k$, and $R=\mathbb{Q}$ if $\operatorname{char} k=0$.

Lemma 2.8. For weights $\lambda, \lambda^{\prime}$, the element $\left\langle\lambda \mid \lambda^{\prime}\right\rangle$ belongs to $R$.

Proof. It suffices to find a $c \in R^{\times}$so that $\left\langle\lambda \mid c \lambda^{\prime}\right\rangle$ is in $R$. If $\lambda^{\prime}$ is a root, we take $c:=2 /\left\langle\lambda^{\prime} \mid \lambda^{\prime}\right\rangle=2 v_{\lambda^{\prime}} h^{\vee}$. Because $\lambda^{\prime}$ is a root, $\left\langle\lambda \mid c \lambda^{\prime}\right\rangle$ is an integer and so in $R$.

If $\lambda^{\prime}$ is in the root lattice, then the conclusion follows from the previous case by bilinearity.

For general $\lambda^{\prime}$, we take $c:=h$. Since $h \lambda^{\prime}$ is in the root lattice, $\left\langle\lambda \mid h \lambda^{\prime}\right\rangle$ is in $R$.

We put $\delta$ for the sum of the positive roots.

Lemma 2.9. Suppose that the representation $\pi: G \rightarrow \mathrm{GL}(V)$ is equivalent to $H^{0}(\lambda)$ or $V(\lambda)$ over the algebraic closure of $k$ for some dominant weight $\lambda$. Then:

1. For $\left\{X_{i}\right\}$ a basis of $\mathfrak{g}$ and $\left\{Y_{i}\right\}$ the dual basis with respect to $K$, we have $\sum \pi\left(X_{i}\right) \pi\left(Y_{i}\right)=$ $\langle\lambda \mid \lambda+\delta\rangle \operatorname{Id}_{V}$, where $\delta$ is the sum of the positive roots.

2. For all $x, y \in \mathfrak{g}$ we have

$$
\operatorname{Tr}(\pi(x) \pi(y))=\frac{\langle\lambda \mid \lambda+\delta\rangle \operatorname{dim} V}{\operatorname{dim} G} K(x, y) .
$$

In the statement, we have abused notation by writing $\pi$ also for the differential $\mathfrak{g} \rightarrow \mathfrak{g l}(V)$ of $\pi$.

Sketch of proof. In case $k$ is algebraically closed of characteristic zero, this result is about an irreducible representation and the claims are part of the usual theory of the quadratic Casimir operator $\sum X_{i} Y_{i} \in U(\mathfrak{g})$ as in, for example, [7, Section VIII.6.4, Cor.] or [15, Th. 2.5].

In case char $k=0$, it suffices to verify the claims over an extension field, for which we take the algebraic closure of $k$.

Now suppose that char $k$ is a prime $p$ and $G$ is split. There is a split group $G_{R}$ and representation $\pi_{R}$, both defined over $R$, whose base change to $k$ is equivalent to $G, \pi$. As $\langle\lambda \mid \lambda+\delta\rangle$ and $(\operatorname{dim} G)^{-1}$ are 
in $R$, the claims amount to certain polynomials over $R$ being zero. Those polynomials are zero over the field of fractions $\mathbb{Q}$ of $R$, so they are also zero over the quotient field $\mathbb{F}_{p}$ and therefore over $k$.

Finally, if char $k$ is prime, again it suffices to verify the claims over the algebraic closure of $k$, where $G$ is split.

\section{The representation $A(\mathfrak{g})$}

Define a map $\mathfrak{g} \otimes \mathfrak{g} \rightarrow \operatorname{End}(\mathfrak{g})$ via $X \otimes Y \mapsto h^{\vee}(\operatorname{ad} X)(\operatorname{ad} Y)+X K(Y,-)$. It is bilinear and so provides a $G$-equivariant linear map $\mathfrak{g} \otimes \mathfrak{g} \rightarrow \operatorname{End}(\mathfrak{g})$. Composing this with (2.3), we find a $G$-equivariant linear $\operatorname{map} S: S^{2}(\mathfrak{g}) \rightarrow \operatorname{End}(\mathfrak{g})$ such that

$$
S(X Y):=h^{\vee} \operatorname{ad}(X) \bullet \operatorname{ad}(Y)+P(X Y),
$$

where $P$ is as in Definition 2.4 and $\bullet$ denotes the Jordan product (2.6).

Since $(\operatorname{ad} X)^{\top}=-\operatorname{ad} X$ for all $X \in \mathfrak{g}$, we find that $S(X Y)$ belongs to $\mathcal{H}(\mathfrak{g})$. Since $S$ is linear in $X$ and in $Y$ and symmetric in the two terms, it extends linearly to all of $S^{2}(\mathfrak{g})$. We set:

$$
A(\mathfrak{g}):=\operatorname{im} S \quad \subseteq \mathcal{H}(\mathfrak{g})
$$

Example 3.3. For $X \in \mathfrak{g}$, we have

$$
\operatorname{Tr}\left(S\left(X^{2}\right)\right)=h^{\vee} K(X, X)+\operatorname{Tr}(X K(X,-))=\left(h^{\vee}+1\right) K(X, X) .
$$

Linearizing this shows that $\operatorname{Tr}(S(X Y))=\left(h^{\vee}+1\right) K(X, Y)$ for $X, Y \in \mathfrak{g}$.

Example 3.4. For $S\left(e_{\mathrm{S}}\right)$, we have $P\left(e_{\mathrm{S}}\right)=\mathrm{Id}_{\mathfrak{g}}$ as in Example 2.5. And by Lemma 2.9(1), $\sum\left(\operatorname{ad} X_{i}\right)\left(\operatorname{ad} Y_{i}\right)=\operatorname{Id}_{\mathfrak{g}}$. Therefore, $S\left(e_{\mathrm{S}}\right)=\left(h^{\vee}+1\right) \operatorname{Id}_{\mathfrak{g}}$.

\section{The split case}

Suppose that $G$ is split: that is, it contains a split maximal torus $T$ defined over $k$. (This is automatic if $k$ is algebraically closed.) Fix a Chevalley basis of $\mathfrak{g}$ with respect to $\mathfrak{h}:=\operatorname{Lie}(T)$ in the sense of [44], [43] or [14, Section XX.2.11]. That is, for each root $\alpha$, define elements $H_{\alpha} \in \mathfrak{h}$ and $X_{\alpha} \in \mathfrak{g}$ so that $X_{\alpha}$ spans the $\alpha$ weight space (for the action of $T$ on $\mathfrak{g}$ ), $\mathfrak{g}=\mathfrak{h} \oplus \bigoplus_{\alpha} k X_{\alpha}$,

$$
\left[H_{\beta}, X_{\alpha}\right]=\beta^{\vee}(\alpha) X_{\alpha} \quad \text { and } \quad\left[X_{\alpha}, X_{-\alpha}\right]=H_{\alpha} .
$$

(This last equation differs by a sign from the one used in [7, Section VIII.2.2].) We note that for any root $\alpha$,

$$
K\left(X_{\alpha}, X_{-\alpha}\right)=K\left(H_{\alpha}, H_{\alpha}\right) / 2=2 v_{\alpha} h^{\vee}
$$

by the formulas in [43, pp. E-14, E-15].

Lemma 3.6. Maintain the notation of the preceding paragraph. Suppose that $\alpha$ and $\beta$ are roots of $G$ such that $\alpha+\beta$ is not a root.

1. If $\langle\alpha \mid \beta\rangle=0$, then $S\left(X_{\alpha} X_{\beta}\right) \neq 0$ in $A(\mathfrak{g})$.

2. Suppose $\langle\alpha \mid \beta\rangle>0$. Then $S\left(X_{\alpha} X_{\beta}\right) \neq 0$ in $A(\mathfrak{g})$ if and only if there are two root lengths and $\alpha$ and $\beta$ are both short.

Proof. Since $X_{\alpha}, X_{\beta}$ commute in $\mathfrak{g}$, so do ad $X_{\alpha}$, ad $X_{\beta}$ in $\operatorname{End}(\mathfrak{g})$. Therefore,

$$
S\left(X_{\alpha} X_{\beta}\right) X_{-\alpha}=\left(v_{\alpha}-\alpha^{\vee}(\beta)\right) h^{\vee} X_{\beta}+\frac{1}{2} K\left(X_{\beta}, X_{-\alpha}\right) X_{\alpha} .
$$


If $\langle\alpha \mid \beta\rangle=0$, then the only nonzero term on the right side of (3.7) is $v_{\alpha} h^{\vee} X_{\beta} \neq 0$, verifying (1). We now prove:

(2') Suppose $\langle\alpha \mid \beta\rangle>0$. Then $S\left(X_{\alpha} X_{\beta}\right) X_{-\alpha} \neq 0$ in $\mathfrak{g}$ if and only if $\alpha$ and $\beta$ are both not long.

If $\alpha=\beta$, then $\alpha^{\vee}(\beta)=2$ and (3.7) equals $2\left(v_{\alpha}-1\right) h^{\vee} X_{\beta}$. This is nonzero if and only if $\alpha$ is not long, verifying $\left(2^{\prime}\right)$ in this case.

If $\alpha \neq \beta$, then (3.7) equals $\left(v_{\alpha}-\alpha^{\vee}(\beta)\right) h^{\vee} X_{\beta}$. If $\alpha$ is not long, then either (a) $\beta$ is long, $v_{\alpha}=\alpha^{\vee}(\beta)$, and (3.7) is zero or (b) $\beta$ is also not long, $\alpha^{\vee}(\beta)=1$, and (3.7) is not zero. If $\alpha$ is long, then $v_{\alpha}=\alpha^{\vee}(\beta)=1$; see, for example, [6, Section VI.1.3]. This completes the verification of $\left(2^{\prime}\right)$.

To complete the proof of the lemma, we assume that $\langle\alpha \mid \beta\rangle>0$ and at least one of $\alpha, \beta$ is long, and verify that $S\left(X_{\alpha} X_{\beta}\right)=0$. Because $S\left(X_{\alpha} X_{\beta}\right) H=0$ for all $H \in \mathfrak{h}$, it remains to evaluate

$$
S\left(X_{\alpha} X_{\beta}\right) X_{-\gamma}=h^{\vee}\left[X_{\beta},\left[X_{\alpha}, X_{-\gamma}\right]\right] \text { for } \gamma \neq \alpha, \beta .
$$

By symmetry, we may assume that $\alpha$ is long, so in the Weyl orbit of the highest root $\widetilde{\alpha}$, and we may even assume that $\alpha=\widetilde{\alpha}$. If any of $\widetilde{\alpha}-\gamma, \beta-\gamma$, or $\widetilde{\alpha}+\beta-\gamma$ is not a root, then (3.8) is zero, as claimed.

For sake of contradiction, suppose that all three are roots. This implies $\beta \neq \widetilde{\alpha}$, for otherwise $\widetilde{\alpha}+\beta-\gamma=2 \widetilde{\alpha}-\gamma$ is a root, whence $\gamma=\widetilde{\alpha}$, a contradiction. Since $\gamma$ and $\widetilde{\alpha}-\gamma$ are roots, $\gamma$ is positive.

Note that if $\rho$ is any root orthogonal to $\widetilde{\alpha}$, then since at least one of $\widetilde{\alpha} \pm \rho$ is not a root, neither can be. Consequently, $\langle\widetilde{\alpha} \mid \gamma\rangle \neq 0$. It follows that $\langle\widetilde{\alpha} \mid \gamma\rangle>0$, since $\widetilde{\alpha}+\gamma$ is not a root and $\widetilde{\alpha} \neq-\gamma$. Now $\widetilde{\alpha}$ is long and $\langle\widetilde{\alpha} \mid \beta\rangle,\langle\widetilde{\alpha} \mid \gamma\rangle$ are positive, so $\widetilde{\alpha}^{\vee}(\beta)=\widetilde{\alpha}^{\vee}(\gamma)=1$, whence $\langle\widetilde{\alpha} \mid \beta-\gamma\rangle=0$, contradicting the hypothesis that $\widetilde{\alpha}+\beta-\gamma$ is a root.

Corollary 3.9. $2 \widetilde{\alpha}$ is not a weight of $A(\mathfrak{g})$.

Proof. The $2 \widetilde{\alpha}$ weight space in $S^{2} \mathfrak{g}$ is spanned by $X_{\widetilde{\alpha}}^{2}$, yet $S\left(X_{\widetilde{\alpha}}^{2}\right)=0$ by Lemma 3.6(2).

\section{The commutative algebra $A(\mathfrak{g})$}

Recall the vector space $A(\mathfrak{g})$ defined in (3.2). Define, for $A, B, C, D \in \mathfrak{g}$ :

$$
\begin{aligned}
S(A B) \diamond S(C D)= & \frac{h^{\vee}}{2}(S(A,(\operatorname{ad} C \bullet \operatorname{ad} D) B)+S((\operatorname{ad} C \bullet \operatorname{ad} D) A, B)) \\
& +\frac{h^{\vee}}{2}(S(C,(\operatorname{ad} A \bullet \operatorname{ad} B) D)+S((\operatorname{ad} A \bullet \operatorname{ad} B) C, D)) \\
& +\frac{h^{\vee}}{2}(S([A, C],[B, D])+S([A, D],[B, C])) \\
& +\frac{1}{4}(K(A, C) S(B, D)+K(A, D) S(B, C)) \\
& +\frac{1}{4}(K(B, C) S(A, D)+K(B, D) S(A, C))
\end{aligned}
$$

in $A(\mathfrak{g})$, where on the right side we have added extra commas in the arguments for the $S$ terms (for example, writing $S(X, Y)$ instead of $S(X Y))$ for clarity.

Lemma 4.2. The formula (4.1) extends to a symmetric bilinear map $\diamond: A(\mathfrak{g}) \times A(\mathfrak{g}) \rightarrow A(\mathfrak{g})$.

Proof. Since both sides of (4.1) are linear in each of $A, B, C, D$ and symmetric under swapping $A, B$ and $C, D$, it remains only to check that $\diamond$ is well defined: that is, that the expression given for $S(A B) \diamond S(v)$ is zero for all $v \in \operatorname{ker} S$. It is sufficient to check this over an algebraic closure of $k$, where we are reduced to the following computation. 
Let $Y, X_{1}, \ldots, X_{r} \in \mathfrak{g}$ be such that $S\left(\sum X_{i}^{2}\right)=0$. The expression for $S\left(Y^{2}\right) \diamond \sum S\left(X_{i}^{2}\right)$ is

$$
\begin{aligned}
& h^{\vee} \sum S\left(\left((\operatorname{ad} Y)^{2} X_{i}\right) X_{i}\right)+h^{\vee} \sum S\left(\left(\left(\operatorname{ad} X_{i}\right)^{2} Y\right) Y\right) \\
& \quad+h^{\vee} \sum S\left(\left[Y, X_{i}\right]\left[Y, X_{i}\right]\right)+\sum K\left(Y, X_{i}\right) S\left(X_{i} Y\right) .
\end{aligned}
$$

As $\sum S\left(X_{i}^{2}\right)=0, \sum P\left(X_{i}^{2}\right)=-h^{\vee} \sum\left(\operatorname{ad} X_{i}\right)^{2}$, so the second and fourth terms in (4.3) cancel.

Furthermore, as $S$ is $\mathfrak{g}$-equivariant, we have

$$
[\operatorname{ad} Z, S(A B)]=S([Z, A] B)+S(A[Z, B]) \quad \text { for } A, B, Z \in \mathfrak{g} \text {. }
$$

Adding the first and third terms in (4.3), dividing by $h^{\vee}$ and applying this identity twice gives

$$
\left[\operatorname{ad} Y, \sum S\left(\left[Y, X_{i}\right] X_{i}\right)\right]=\frac{1}{2}\left[\operatorname{ad} Y,\left[\operatorname{ad} Y, \sum S\left(X_{i}^{2}\right)\right]\right]=0 .
$$

In summary, (4.3) is zero. Therefore, if we write $a, a^{\prime} \in A(\mathfrak{g})$ as $a=S(w)$ and $a^{\prime}=S\left(w^{\prime}\right)$ for $w, w^{\prime} \in \mathrm{S}^{2} \mathfrak{g}$, the value of $a \diamond a^{\prime}$ given by (4.1) does not depend on the choice of $w, w^{\prime}$.

With Lemma 4.2 in hand, we view $A(\mathfrak{g})$ as a commutative $k$-algebra with the product $\diamond$ defined by (4.1).

Lemma 4.5. The identity transformation e of $\mathfrak{g}$ is the multiplicative identity in $A(\mathfrak{g}):$ that is, $e \diamond a=a$ for all $a \in A(\mathfrak{g})$.

Proof. First note that $e$ is in $A(\mathfrak{g})$ by Example 3.4. We may enlarge our base field and so assume that $k$ is algebraically closed and in particular that $\mathfrak{g}$ has an orthonormal basis $\left\{X_{i}\right\}$. Combining (4.1) and (4.4), we obtain

$$
S\left(X_{i}^{2}\right) \diamond S\left(Y^{2}\right)=\frac{h^{\vee}}{2}\left[\operatorname{ad} Y,\left[\operatorname{ad} Y, S\left(X_{i}^{2}\right)\right]\right]+h^{\vee} S\left(\left(\operatorname{ad} X_{i}\right)^{2} Y, Y\right)+K\left(X_{i}, Y\right) S\left(X_{i} Y\right) .
$$

If we sum both sides over $i$, we have $\left(h^{\vee}+1\right) e \diamond S\left(Y^{2}\right)$ on the left by Example 2.5 and $0+h^{\vee} S\left(Y^{2}\right)+S\left(Y^{2}\right)$ on the right. Consequently $S\left(Y^{2}\right) \diamond e=S\left(Y^{2}\right)$, as required.

Remark 4.6. The paper [11] constructs an algebra $A$ similar to $A(\mathfrak{g})$ that is also a subspace of $\mathcal{H}(\mathfrak{g})$, but with a different product, which we denote by $*$ for the moment. It defines $a * a^{\prime}:=\operatorname{proj}_{A}\left(a \bullet a^{\prime}\right)$, which differs from our product defined in (4.1). The analog of (4.1) for their multiplication $*$ has additional terms. For the case where $G$ has type $E_{8}$, both algebras can be viewed as different ways of adding a unit to the irreducible 3875-dimensional representation. Since that representation supports a unique $E_{8}$-invariant product, the difference between our multiplications is necessarily minor. That is, if our $A(\mathfrak{g})$ is written as $\mathcal{U}(V, f)$ in the notation of Appendix A, then theirs is $\mathcal{U}(V, c f)$ for some invertible $c \neq 1$ in $k$.

\section{A Jordan subalgebra}

Suppose that $\mathfrak{I}$ is an abelian subalgebra of $\mathfrak{g}$. (For example, one could take $\mathfrak{l}=\mathfrak{h}$.) Define a $k$-linear map

$$
i: P\left(\mathrm{~S}^{2} \mathfrak{l}\right) \rightarrow A(\mathfrak{g}) \quad \text { via } \quad i(P(x y)):=S(x y) .
$$

Writing out (4.1), we find that

$$
i(P(x y) \bullet P(z w))=S(x y) \diamond S(z w) .
$$


That is, $i$ is an algebra homomorphism, and the image of $P\left(\mathrm{~S}^{2} \mathfrak{l}\right)$ is a Jordan subalgebra of $A(\mathfrak{g})$. (Note that the identity element of $P\left(S^{2} \mathfrak{l}\right)$ need not map to the identity element of $A(\mathfrak{g})$; see the proof of Proposition 5.3.)

Lemma 4.8. If $\mathfrak{I}$ is an abelian subalgebra of $\mathfrak{g}$ and the Killing form $K$ restricts to be nondegenerate on $\mathfrak{I}$, then the homomorphism (4.7) is injective.

Note that when $\left.K\right|_{\mathfrak{l}}$ is nondegenerate, the isomorphism $\mathfrak{g} \otimes \mathfrak{g} \stackrel{\sim}{\rightarrow} \mathfrak{g} \otimes \mathfrak{g}^{*}$ restricts to an isomorphism $\ell \otimes \ell \stackrel{\sim}{\rightarrow} \ell \otimes \ell^{*}$ which identifies $P\left(\mathrm{~S}^{2} \mathfrak{I}\right)$ with the Jordan algebra $\mathcal{H}(\mathfrak{I})$ of symmetric elements in End(I).

Proof. The definition of $S$ shows that $i\left(P\left(\mathrm{~S}^{2} \mathfrak{l}\right)\right)$, as a subspace of $\operatorname{End}(\mathfrak{g})$, acts on $\mathfrak{I}$ via $i\left(P\left(X^{2}\right)\right)(Y)=$ $P\left(X^{2}\right)(Y)$ for all $X, Y \in \mathfrak{I}$. The nondegeneracy of $K$ then identifies $i\left(P\left(\mathrm{~S}^{2} \mathfrak{I}\right)\right)$ with the symmetric elements in $\operatorname{End}(\mathfrak{l})$.

Example 4.9. Suppose $G$ is split and not of type $A_{1}$ or $A_{2}$. Fix a Chevalley basis for $G$ as in Section 3. For $H \in \mathfrak{h}$ such that $K(H, H)$ is not zero, the element $u_{H}:=i\left(P\left(H^{2}\right)\right) / K(H, H)$ is an idempotent in $A(\mathfrak{g})$. This provides an idempotent in $A(\mathfrak{g})$ for every element of $\mathbb{P}(\mathfrak{h})$ in the complement of the quadric hypersurface defined by $K(X, X)=0$. Clearly, if $k$ is infinite, there are infinitely many idempotents in $A(\mathfrak{g})$.

Now, there is a positive root $\gamma$ that is orthogonal to the highest root $\widetilde{\alpha}$. For the element $S\left(X_{\widetilde{\alpha}} X_{\gamma}\right)$, which is nonzero by Lemma 3.6(1), we have

$$
u_{H} \diamond S\left(X_{\widetilde{\alpha}} X_{\gamma}\right)=\lambda_{H} S\left(X_{\widetilde{\alpha}} X_{\gamma}\right) \quad \text { for } \quad \lambda_{H}=\frac{h^{\vee}((\widetilde{\alpha}+\gamma)(H))^{2}}{2 K(H, H)} .
$$

The map $H \mapsto \lambda_{H}$ is a rational function $\mathfrak{h} \rightarrow k$ that is not constant and therefore is dominant. In particular, the collection of eigenvalues of the maps $x \mapsto u \diamond x$ as $u$ varies over the idempotents of $A(\mathfrak{g})$ is not contained in $\left\{0, \frac{1}{2}, 1\right\}$, and therefore $A(\mathfrak{g})$ is not power-associative [39, Ch. V].

\section{5. $A(\mathfrak{g})$ as an algebra obtained by adding a unit}

The usual trace form $\operatorname{Tr}: \operatorname{End}(\mathfrak{g}) \rightarrow k$ is linear and $G$-invariant. We use it to define a counit, in the sense of the appendix, as $\varepsilon:=\frac{1}{\operatorname{dim} G} \operatorname{Tr}$ so that $\varepsilon(e)=1$, for $e=\operatorname{Id}_{\mathfrak{g}}$ the identity element in $A(\mathfrak{g})$ (Lemma 4.5). Thus we obtain a bilinear form $\tau$ on $A(\mathfrak{g})$ via (A.5), $\tau\left(a, a^{\prime}\right):=\varepsilon\left(a \diamond a^{\prime}\right)$. The form $\tau$ is evidently $G$-invariant (because $\operatorname{Tr}$ and $\diamond$ are), symmetric (because $\diamond$ is commutative), and bilinear.

Example 5.1. For $X, Y \in \mathfrak{g}$, Example 3.3 gives

$$
\tau(e, S(X Y))=\frac{h^{\vee}+1}{\operatorname{dim} G} K(X, Y) \quad \text { for } X, Y \in \mathfrak{g} .
$$

We also note for future reference:

$$
\begin{aligned}
\tau\left(S\left(X^{2}\right), S\left(Y^{2}\right)\right) & =\left(\frac{h^{\vee}+1}{\operatorname{dim} G}\right)\left(-h^{\vee} K([X, Y],[X, Y])+K(X, Y)^{2}\right) \\
& =\left(\frac{h^{\vee}+1}{\operatorname{dim} G}\right) K\left(S\left(X^{2}\right) Y, Y\right) .
\end{aligned}
$$

Using the counit $\varepsilon$ defined above, the algebra $A(\mathfrak{g})$ can be viewed as an algebra $\mathcal{U}(V, f)$ as in the appendix, where $V$ is the vector space ker $\varepsilon$ endowed with the commutative product $\cdot$ and $f$ as defined in (A.4). With this notation, we prove:

Proposition 5.3. If $G$ is not of type $A_{1}$ nor $A_{2}$, then:

1. The multiplication on $V$ is not zero.

2. Neither Vnor $U(V, c f)$ is power-associative for any $c \in k$. 
For the excluded cases of $A_{1}$ and $A_{2}$; see Examples 7.1 and 10.9 respectively.

Proof. For each claim, we may enlarge $k$ and so assume that the Lie algebra $\mathfrak{h}$ of some maximal torus in $G$ has an orthonormal basis $X_{1}, \ldots, X_{\ell}$. We set $B:=i\left(P\left(S^{2} \mathfrak{h}\right)\right)$.

We begin with (1). By (5.2), for $i \neq j, S\left(X_{i} X_{j}\right)$ is in $V$. On the other hand, if $\ell \geq 3$,

$$
S\left(X_{1} X_{2}\right) \diamond S\left(X_{1} X_{3}\right)=i\left(P\left(X_{1} X_{2}\right) \bullet P\left(X_{1} X_{3}\right)\right)=\frac{1}{4} S\left(X_{2} X_{3}\right) \neq 0
$$

and we are done. If $\ell=2$, then $e^{\prime}:=S\left(X_{1}^{2}+X_{2}^{2}\right)$ is the identity element in $B$ by Example 2.7, yet

$$
s:=\tau\left(e, e^{\prime}\right)=2 \frac{h^{\vee}+1}{\operatorname{dim} G}=\frac{h^{\vee}+1}{h+1}
$$

is not 1 because $G$ is not of type $A_{2}$. Then $e^{\prime}-s e$ is in $V$ and $\left(e^{\prime}-s e\right) \cdot S\left(X_{1} X_{2}\right)=(1-s) S\left(X_{1} X_{2}\right) \neq 0$, verifying (1).

For (2), put $r:=\left(h^{\vee}+1\right) /(\operatorname{dim} G)$, a rational number whose denominator is not divisible by char $k$. Since $h^{\vee} \leq h, 0<r \leq 1 / 2$. Define a map $S^{+}: S^{2} \mathfrak{g} \rightarrow V$ by $S^{+}(p)=S(p)-\varepsilon(S(p)) e$. Applying Example 5.1, we find:

$$
\tau\left(S^{+}\left(X^{2}\right), S^{+}\left(X^{2}\right)\right)=r(1-r) K(X, X)^{2} \quad \text { for } X \in \mathfrak{g} .
$$

Therefore $\tau$ (equivalently, $f$ ) is not zero on $V$, and in particular $f$ is not alternating.

Set $b:=i\left(P\left(X_{1}^{2}\right)+t P\left(X_{2}^{2}\right)\right)$, where $t \in k$ is neither 0 nor 1 , so $(1,0), b$, and $b^{2}=i\left(P\left(X_{1}^{2}\right)+t^{2} P\left(X_{2}^{2}\right)\right)$ are linearly independent (Lemma 4.8). Let $B$ be the subalgebra of $\mathcal{U}(V, f)$ generated by $(1,0)$ and $b$. Then $B=\mathcal{U}\left(V \cap B,\left.f\right|_{V \cap B}\right)$, and $B$ is power-associative because $b$ generates a Jordan subalgebra of $\mathcal{U}(V, f)$.

We have already observed in Example 4.9 that $\mathcal{U}(V, f)$ itself is not power-associative, so we fix $c \neq 1$. By Proposition A.13, $\mathcal{U}\left(V \cap B,\left.c f\right|_{V \cap B}\right)$ is not strictly power-associative, and so $\mathcal{U}(V, c f)$ is not strictly power-associative either. It follows that $\mathcal{U}(V, c f)$ is not power-associative, because char $k \neq 2,3,5$ and $\mathcal{U}(V, c f)$ is commutative. The case $c=0$ gives that $V$ itself is not power-associative.

As opposed to defining the product on $A(\mathfrak{g})$ via (4.1), one could build $A(\mathfrak{g})$ 'from below' by starting with a $G$-invariant commutative product - on a representation $V$ and a $G$-invariant bilinear form $f$ and setting $A(\mathfrak{g})$ to be $\mathcal{U}(V, f)$. In case $G$ has type $E_{8}$ and $V$ is the irreducible 3875-dimensional representation, both $\cdot$ and $f$ are uniquely determined up to a factor in $k^{\times}$. But only the scalar factor on $f$ matters (Remark A.3), and (2) says that the resulting algebra is not power-associative, no matter what choice one makes for that parameter.

Similarly, the conclusion of Lemma 6.1 below would be unchanged by multiplying $f$ by a scalar factor, as is clear from Proposition A.7.

\section{Associativity of the bilinear form $\tau$}

The following property of the symmetric bilinear form $\tau$ on $A(\mathfrak{g})$ is sometimes described as saying that ' $\tau$ is associative', especially in the context of Dieudonné's lemma as in [22, pp. 199, 239].

Lemma 6.1. The bilinear form $\tau$ on $A(\mathfrak{g})$ satisfies

$$
\tau\left(a \diamond a^{\prime}, a^{\prime \prime}\right)=\tau\left(a, a^{\prime} \diamond a^{\prime \prime}\right) \quad \text { for all } a, a^{\prime}, a^{\prime \prime} \in A(\mathfrak{g}) .
$$

Proof. It suffices to verify this in the case $a=S\left(X^{2}\right), a^{\prime}=S\left(Y^{2}\right)$, and $a^{\prime \prime}=S\left(Z^{2}\right)$ for $X, Y, Z \in \mathfrak{g}$. 
Expanding out following the definitions, one finds:

$$
\begin{aligned}
\left(\frac{\operatorname{dim} G}{h^{\vee}+1}\right) \tau\left(a \diamond a^{\prime}, a^{\prime \prime}\right) \\
=\left(h^{\vee}\right)^{2}\left(K\left((\operatorname{ad} Z)^{2} Y,(\operatorname{ad} X)^{2} Y\right)+K\left((\operatorname{ad} Z)^{2} X,(\operatorname{ad} Y)^{2} X\right)+K\left((\operatorname{ad} Z)^{2}[X, Y],[X, Y]\right)\right) \\
\quad+h^{\vee}\left(K(Z, Y) K\left(Z,(\operatorname{ad} X)^{2} Y\right)+K(X, Y) K\left((\operatorname{ad} Z)^{2} Y, X\right)+K(Z, X) K\left(Z,(\operatorname{ad} Y)^{2} X\right)\right) \\
\quad+h^{\vee} K([X, Y], Z)^{2}+K(X, Y) K(X, Z) K(Y, Z) .
\end{aligned}
$$

Put $\psi$ for the alternating trilinear form $\psi(A, B, C)=K([A B], C)$ on $\mathfrak{g}$ and observe that $\Psi:=$ $\psi([X, Z],[X, Y],[Y, Z])$ is invariant under permutations of the variables $X, Y, Z$. We have:

$$
\begin{aligned}
\Psi & =K([X, Z],[[[X, Y], Y], Z])+K([X, Z],[Y,[[X, Y], Z]]) \\
& =-K\left((\operatorname{ad} Z)^{2} Y,(\operatorname{ad} Y)^{2} X\right)-K([[X, Z], Y],[Z,[X, Y]]) .
\end{aligned}
$$

Adding this equation to the same equation with $X$ and $Y$ swapped gives that $-2 \Psi$ is the first term in parentheses on the right side of (6.3). That is,

$$
\left(\frac{\operatorname{dim} G}{h^{\vee}+1}\right) \tau\left(a \diamond a^{\prime}, a^{\prime \prime}\right)=-2\left(h^{\vee}\right)^{2} \Psi-h^{\vee} E+h^{\vee} \psi(X, Y, Z)^{2}+K(X, Y) K(X, Z) K(Y, Z)
$$

with

$$
E=\psi(X, Y,[X, Z]) K(Y, Z)+\psi(X, Z,[Y, Z]) K(X, Y)+\psi(Y, X,[Y, Z]) K(X, Z) .
$$

Each of the four terms on the right side of (6.4) is unchanged when we swap $X$ and $Z$, and therefore the claim is verified.

Remark 6.5. Here is another argument to show associativity of $\tau$ that works when $G$ has type $E_{8}$. In that case, $A(\mathfrak{g})=k e \oplus V$, where $V$ is an irreducible representation of $G$ (Lemma 7.2), the restriction $f$ of $\tau$ to $V$ is nondegenerate, and the space $\left(V^{*} \otimes V^{*} \otimes V^{*}\right)^{G}$ of $G$-invariant trilinear forms on $V$ is 1-dimensional. It follows then that the linear maps defined by sending $v \otimes v^{\prime} \otimes v^{\prime \prime} \in \otimes^{3} V$ to $f\left(v \cdot v^{\prime}, v^{\prime \prime}\right)$ and $f\left(v, v^{\prime} \cdot v^{\prime \prime}\right)$ agree up to a scalar factor, where $f$ is the restriction of $\tau$ to $V$. The two cubic forms are nonzero (Proposition 5.3(1)) and agree when $v=v^{\prime}=v^{\prime \prime}$ is a generic element of $V$, so the two forms agree in general: that is, $f$ is associative with respect to the product ', whence $\tau$ is associative with respect to the product $\diamond$ on $A(\mathfrak{g})$ by Proposition A.7.

\section{7. $\mathbf{A}(\mathfrak{g})$ as a representation of $\mathbf{G}$}

The counit $\varepsilon$ gives a direct sum decomposition $A(\mathfrak{g})=k e \oplus V$ as a representation of $G$. In this section, we describe $V$ as a representation of $G$ and show that its dimension and character depend only on the root system of $G$ and not on the field $k$ nor even the characteristic of $k$. We use the notion of a Weyl module recalled in Section 2.

Example 7.1 $\left(A\left(\mathfrak{s l}_{2}\right)\right)$. Suppose $G$ is split of type $A_{1}$, so $\mathfrak{g}=\mathfrak{s l}_{2}$. By hypothesis, char $k$ is zero or at least 5, so the Weyl module $V(4)$ of $G$ with highest weight 4 is irreducible over $k$ [49]. It is a submodule of $\mathcal{H}(\mathfrak{g})$ generated by $P\left(X_{\widetilde{\alpha}}^{2}\right)$ and $\mathcal{H}(\mathfrak{g}) / k$ is $V(4)$ by dimension count. As $A(\mathfrak{g})$ does not meet $V(4)$ (Corollary 3.9), it follows that $A\left(\mathfrak{s l}_{2}\right)=k$ as a vector space, spanned by $\operatorname{Id}_{\mathfrak{g}}$ : that is, $A\left(\mathfrak{s l}_{2}\right)$ is identified with $k$ as a $k$-algebra.

The notion of a Weyl module still makes sense when $G$ is not assumed to be split. In that case, one still picks a maximal torus $T$ defined over $k$. Pick any Borel subgroup $B$ of $G \times \bar{k}$ containing $T$; equivalently, pick a cone of dominant weights in the character lattice $T^{*}$. There is a natural action of the Galois group $\operatorname{Aut}(\bar{k} / k)$ on $T^{*}$, which maps the cone to itself if and only if $B$ is defined over $k$. In 
Table 1. Data for some exceptional groups $G$. The fundamental dominant weights in the formula for $\lambda$ are numbered as in [6].

\begin{tabular}{lcccccc}
\hline Type of $G$ & $A_{2}$ & $G_{2}$ & $F_{4}$ & $E_{6}$ & $E_{7}$ & $E_{8}$ \\
\hline Dual Coxeter number $h^{\vee}$ & 3 & 4 & 9 & 12 & 18 & 30 \\
Coxeter number $h$ & 3 & 6 & 12 & 12 & 18 & 30 \\
Dominant weight $\lambda$ & $\omega_{1}+\omega_{2}$ & $2 \omega_{1}$ & $2 \omega_{4}$ & $\omega_{1}+\omega_{6}$ & $\omega_{6}$ & $\omega_{1}$ \\
Dim. of irred. rep. $L(\lambda)$ & 8 & 27 & 324 & 650 & 1539 & 3875 \\
\hline
\end{tabular}

any case, there is a canonical way to modify the action using the Weyl group to produce a new action of $\operatorname{Aut}(\bar{k} / k)$ on $T^{*}$ that does leave the cone invariant; see [4, 6.2] or [46, Section 3.1]. (This action permutes the simple roots and is determined by how it does so, and therefore is equivalent to an action of $\operatorname{Aut}(\bar{k} / k)$ on the Dynkin diagram of $G$.)

Suppose that $\lambda \in T^{*}$ is a dominant weight, is in the root lattice, and is fixed by the action of $\operatorname{Aut}(\bar{k} / k)$ on the dominant weights. (This holds, for example, for $\lambda=2 \widetilde{\alpha}$ and any $G$, or for $G$ and $\lambda$ as in Table 1.) Then there is a unique representation of $G$ over $k$ that becomes isomorphic to $V(\lambda)$ (respectively, $H^{0}(\lambda)$; respectively, $L(\lambda)$ ) over $\bar{k}$. This is proved in [46, Th. 3.3] for the irreducible $L(\lambda)$, and the same argument works for the other two representations. Therefore, for such a $\lambda$, it makes sense to also use the same notation for the representation of $G$ over $k$.

Proposition 7.2. Suppose char $k=0$,

$$
\operatorname{char} k \geq\left(\begin{array}{c}
\operatorname{dim} G+1 \\
2
\end{array}\right) /(\operatorname{rank} G)
$$

or $G$ is as in Table 1. As a representation of $G, A(\mathfrak{g})$ is a direct sum of pairwise non-isomorphic irreducible modules and $\mathcal{H}(\mathfrak{g})=A(\mathfrak{g}) \oplus V(2 \widetilde{\alpha})$. Furthermore, if $G$ and $\lambda$ are as in Table 1 , then $A(\mathfrak{g})=k \oplus L(\lambda)$.

Note that the displayed lower bound on char $k$ grows like $(\operatorname{rank} G)^{3}$, so it is somewhat more restrictive than our global hypothesis that char $k=0$ or at least $h+2$, because $h+2$ grows like $\operatorname{rank} G$.

Proof of Proposition 7.2. We first address the case where $k$ is algebraically closed of characteristic zero. Then $\mathcal{H}(\mathfrak{g}) \cong k \oplus J \oplus L(2 \widetilde{\alpha})$, where $k$ is the span of $e$ and $L(2 \widetilde{\alpha})$ is the $G$-submodule generated by $P\left(X_{\widetilde{\alpha}}^{2}\right)$, which does not belong to $A(\mathfrak{g})$ by Corollary 3.9. Writing $J$ as a sum of irreducible representations $\oplus_{i} L\left(\lambda_{i}\right)$, the values of $\lambda_{i}$ are known. If $G$ is from Table 1 , then $J=L(\lambda)$ is described in [10], where it is denoted by $Y_{2}^{*}$. If $G$ has type $A_{1}$, then $J=0$. Otherwise, $J$ is a sum of three irreducible components for type $D_{4}$ or two for the other types; see [48] and [30] for more on this decomposition and related subjects. In all cases, the $\lambda_{i}$ are distinct, are not zero and are maximal weights for $J$.

To complete the proof for this $k$, we must verify that $J \subseteq A(\mathfrak{g})$. The bulk of the $\lambda_{i}$ s are of the form $\widetilde{\alpha}+\beta$ for a root $\beta$ obtained by the following procedure. Take the Dynkin diagram for $G$, delete all simple roots that are not orthogonal to the highest root $\widetilde{\alpha}$, and select one of the connected components that remains. It corresponds to a subsystem of the root system of $G$ and is the subsystem for a subalgebra $\mathfrak{g}^{\prime}$ of $\mathfrak{g}$ normalized by our chosen maximal torus $T$. (One says that $\mathfrak{g}^{\prime}$ is a regular subalgebra.) Put $\beta$ for the highest root of $\mathfrak{g}^{\prime}$ (in the ordering induced from the chosen ordering on the weights of $G$ ). The element $S\left(X_{\widetilde{\alpha}} X_{\beta}\right)$ is not zero by Lemma 3.6(1), so we conclude that $S\left(X_{\widetilde{\alpha}} X_{\beta}\right)$ is a highest weight vector and $L(\widetilde{\alpha}+\beta) \subseteq A(\mathfrak{g})$.

For types $A_{n}$ and $C_{n}$ with $n \geq 2$, one component of $J$ is of the form considered in the previous paragraph (and so we have shown that it belongs to $A(\mathfrak{g})$ ) and the other is $\lambda$ for $\lambda$ the highest short root. 
For type $A_{n}$, we set

$$
\begin{gathered}
\beta_{j}:=\alpha_{1}+\alpha_{2}+\cdots+\alpha_{j} \quad \text { for } 1 \leq j<n, \\
\gamma_{j}:=\alpha_{j}+\alpha_{j+1}+\cdots+\alpha_{n} \quad \text { for } 1<j \leq n, \text { and } \\
p:=2 h^{\vee} X_{\widetilde{\alpha}} H_{\omega_{1}-\omega_{n}}^{\prime}-\sum_{j=1}^{n-1}\left[X_{\widetilde{\alpha}}, X_{-\beta_{j}}\right] X_{\beta_{j}}+\sum_{j=2}^{n}\left[X_{\widetilde{\alpha}}, X_{-\gamma_{j}}\right] X_{\gamma_{j}} \in \mathrm{S}^{2} \mathfrak{g},
\end{gathered}
$$

where the $\alpha_{i}$ are the simple roots as numbered in [6] and $\omega_{i}$ is the corresponding fundamental dominant weight. For type $C_{n}, \lambda=\widetilde{\alpha}-\beta$ for $\beta$ the simple root not orthogonal to $\widetilde{\alpha}$, and we set

$$
p:=2 X_{\widetilde{\alpha}} X_{-\beta}-\sum_{\mu \in \Phi_{S}}\left[X_{-\mu}, X_{\widetilde{\alpha}}\right]\left[X_{\mu}, X_{-\beta}\right] \quad \in \mathrm{S}^{2} \mathfrak{g}
$$

for $\Phi_{S}$ the set of short roots. In either case, $p$ has weight $\lambda$, and a lengthy verification shows that $S(p)$ is not zero and is fixed by each unipotent subgroup of $G$ corresponding to a positive root, verifying that $\lambda$ is a highest weight vector in $A(\mathfrak{g})$ and therefore that $L(\lambda)$ is a summand of $A(\mathfrak{g})$ and completing the proof for this $k$.

Next suppose that $k$ is algebraically closed of characteristic $p \neq 0$. We transfer the results proved over $\mathbb{C}$ to $k$ via $R:=\mathbb{Z}_{(p)}$. We use subscripts $\mathbb{C}, k, R$ to denote corresponding objects over these three rings. For example, let $G_{R}$ denote the unique split reductive group scheme over $R$ with the same root datum as $G$, so $G_{R} \times k \cong G$, and put $\mathfrak{g}_{R}:=\operatorname{Lie}\left(G_{R}\right)$. For each dominant weight $\eta \in T^{*}$, there is a Weyl module $V_{R}(\eta)$ of $G_{R}$ defined over $R$ such that $L_{\mathbb{C}}(\eta)=V_{R}(\eta) \times \mathbb{C}$ and $V_{k}(\eta)=V_{R}(\eta) \times k$.

The representations $V_{k}\left(\lambda_{i}\right)$ and $V_{k}(2 \widetilde{\alpha})$ are irreducible. If $G$ is from Table 1 , then this fact is contained in the tables in [31]. Otherwise, $p \geq(\operatorname{dim} \mathcal{H}(g)) /(\operatorname{rank} G)$, and every representation of $G$ of dimension at most that of $\mathcal{H}(\mathfrak{g})$ is semisimple [32, Cor. 1.1.1]. A semisimple Weyl module is irreducible [23, Cor. II.2.3], proving the claim.

The map $S: S^{2} \mathfrak{g} \rightarrow \mathcal{H}(\mathfrak{g})$ is defined over $R$, and the dimension of its image over $\mathbb{C}$ is at least as large as its image over $k$ by upper semicontinuity of dimension. As the arguments above show that the irreducible representation $L_{k}\left(\lambda_{i}\right)$ belongs to $A(\mathfrak{g})$ over $k$ for all $i$ and there are no nontrivial extensions among the $L_{k}\left(\lambda_{i}\right)$ [23, II.4.13], we conclude that $A(\mathfrak{g}) \cong k \oplus\left(\oplus_{i} L_{k}\left(\lambda_{i}\right)\right)$ as a representation of $G$.

As a quotient of vector spaces, $\mathcal{H}(\mathfrak{g}) / A(\mathfrak{g})$ is a representation of $G$ with highest weight $2 \widetilde{\alpha}$, so there is a nonzero homomorphism $V_{k}(2 \widetilde{\alpha}) \rightarrow \mathcal{H}(\mathfrak{g}) / A(\mathfrak{g})$. The preceding arguments showed that the dimension of $A(\mathfrak{g})$, and hence the dimensions of both the domain and codomain of the map do not depend on $k$. Since $V_{k}(2 \widetilde{\alpha})$ is irreducible, the map is injective and so an isomorphism by dimension count. There are no non-trivial extensions among the irreducible representations appearing in the composition series for $\mathcal{H}(\mathfrak{g})$, whence the claim in the second sentence of the proposition.

Finally, drop the hypothesis that $k$ is algebraically closed; in particular, $G$ need not be split. The center of $G$ acts trivially on $\mathcal{H}(\mathfrak{g})$, so we may assume that $G$ is adjoint. We view $G$ and the representation $A(\mathfrak{g})$ as being obtained from a representation $A\left(\mathfrak{g}_{0}\right)$ of the unique split form $G_{0}$ of $G$ over $k$ by twisting by a 1 -cocycle $\eta$ in Galois cohomology $Z^{1}\left(\operatorname{Aut}(\bar{k} / k)\right.$, Aut $\left.\left(G_{0}\right)\right)$ as in [40, Section III.1.3]. (Recall that the component group of $\operatorname{Aut}\left(G_{0}\right)$ can be identified with the automorphism group of the Dynkin diagram as in [14, Ch. XXIV, 1.3, 3.6, 5.6] or [42, Section 16.3], and the image of $\eta$ in $Z^{1}\left(\operatorname{Aut}(\bar{k} / k), \operatorname{Aut}\left(G_{0}\right) / \operatorname{Aut}\left(G_{0}\right)^{\circ}\right)$ encodes the $*$-action.) If $G$ is not of type $D_{4}$, then the $\lambda_{i}$ s are each fixed by the $*$-action and belong to the root lattice; hence, each representation $L\left(\lambda_{i}\right)$ of $G_{0}$ is naturally compatible with the twisting by $\eta$, giving an irreducible representation of $G$ defined over $k$, as discussed before the statement of the proposition. For $G$ of type $D_{4}, A\left(\mathfrak{g}_{0}\right)=k \oplus L\left(\lambda_{1}\right) \oplus L\left(\lambda_{2}\right) \oplus L\left(\lambda_{3}\right)$ as a representation of $G_{0}$, and the $*$-action permutes the $\lambda_{i}$ s according to its action on the three terminal vertices in the Dynkin diagram. As in [46, Th. 7.2], we find that the representation $A(\mathfrak{g}) / k$ of $G$, which is obtained by twisting the representation $A\left(\mathfrak{g}_{0}\right) / k$ of $G_{0}$ by $\eta$, is a sum of $o$ distinct irreducible representations of $G$ over $k$, where $o$ is the number of orbits of $\operatorname{Aut}(\bar{k} / k)$ on the set $\left\{\lambda_{1}, \lambda_{2}, \lambda_{3}\right\}$. 
For $G$ as in Table $1, \operatorname{dim}_{k} A(\mathfrak{g})=1+\operatorname{dim} L(\lambda)$ as provided in the table. For $G$ of type $A, B, C$, or $D$ and under the hypotheses of Proposition 7.2, we have

$$
\operatorname{dim}_{k} A(\mathfrak{g})=\left(\begin{array}{c}
\operatorname{dim} G+1 \\
2
\end{array}\right)-\operatorname{dim} V(2 \widetilde{\alpha}),
$$

where $\operatorname{dim} V(2 \widetilde{\alpha})$ is given by the Weyl dimension formula.

\section{8. $\tau$ is nondegenerate and $A(\mathfrak{g})$ is simple}

Proposition 8.1. $\tau$ is nondegenerate on $A(\mathfrak{g})$.

Some authors would summarize Lemma 6.1 and Proposition 8.1, which say that $A(\mathfrak{g})$ has an associative and nondegenerate symmetric bilinear form, by saying ' $A(\mathfrak{g})$ is metrized'.

The proof leverages the following.

Example 8.2. Suppose we are in the situation of Lemma 3.6(1): that is, $G$ is split and $\alpha, \beta$ are orthogonal roots and $\alpha+\beta$ is not a root. Recall from (3.5) that $K\left(X_{\gamma}, X_{-\gamma}\right)$ is not zero in $k$ for every root $\gamma$ (and is positive when $k \subseteq \mathbb{R}$ ) and that $S\left(X_{\alpha} X_{\beta}\right) X_{-\alpha}=v_{\alpha} h^{\vee} X_{\beta}$. Bilinearizing Example 5.1, we have

$$
\begin{aligned}
\tau\left(S\left(X_{\alpha} X_{\beta}\right), S\left(X_{-\alpha} X_{-\beta}\right)\right) & =\left(\frac{h^{\vee}+1}{\operatorname{dim} G}\right) K\left(S\left(X_{\alpha} X_{\beta}\right) X_{-\alpha}, X_{-\beta}\right) \\
& =\left(\frac{h^{\vee}+1}{\operatorname{dim} G}\right) 2\left(h^{\vee}\right)^{2} v_{\alpha} v_{\beta},
\end{aligned}
$$

where the second equality is by (3.5). Note that this is not zero in $k$. Moreover, in case $k \subseteq \mathbb{R}$, the expression is positive.

Proof of Proposition 8.1. We may enlarge $k$ and so assume that $G$ is split. Recall from Section 7 that $A(\mathfrak{g})=k e \oplus\left(\oplus_{i} L\left(\lambda_{i}\right)\right)$ for a set of dominant weights $\left\{\lambda_{i}\right\}$. This sum is an orthogonal sum with respect to $\tau$, and therefore it suffices to verify the claims for the restriction of $\tau$ to each $L\left(\lambda_{i}\right)$.

Pick $p \in \mathrm{S}^{2} \mathrm{~g}$ such that $S(p)$ is a highest weight vector in $L\left(\lambda_{i}\right)$. In case $\lambda_{i}=\widetilde{\alpha}+\beta$ for some positive root $\beta$ orthogonal to $\widetilde{\alpha}$, we take $p:=S\left(X_{\widetilde{\alpha}} X_{\beta}\right)$. Otherwise, $\lambda$ is the highest short root and $G$ has type $A_{n}$ for $n \geq 3$ or $C_{n}$ for $n \geq 2$; in that case, we take $p$ to be as in the proof of Proposition 7.2.

Define $\theta$ to be the automorphism of $\mathfrak{g}$ such that $\left.\theta\right|_{\mathfrak{h}}=-1$ and $\theta\left(X_{\gamma}\right)=X_{-\gamma}$ for each root $\gamma$. Then $S(\theta p)$ is a lowest weight vector in $L\left(\lambda_{i}\right)$. We verify that $\tau(S(p), S(\theta p))$ is not zero; in the first case this is Example 8.2, and in the second case a calculation is required. Therefore, the restriction of $\tau$ to $L\left(\lambda_{i}\right)$ is not zero, so it is nondegenerate, verifying the claim.

Corollary 8.3. If $k=\mathbb{R}$ and $G$ is compact, then $\tau$ is positive-definite on $A(\mathfrak{g})$.

Proof. We continue the notation of the proof of Proposition 8.1. We view $\mathfrak{g}$ as the subalgebra of the split complex Lie algebra consisting of elements fixed by the Cartan involution obtained by composing $\theta$ with complex conjugation as in [7, Section IX.3.2]. Then $v:=p+\theta p$ is in $S^{2} \mathfrak{g}, S(v)$ is in $L\left(\lambda_{i}\right)$, and $\tau(S(v), S(v))=2 \tau(S(p), S(\theta p))>0$. As $G$ is compact, every nonzero $G$-invariant bilinear form on $L\left(\lambda_{i}\right)$ is definite, so $\tau$ is positive definite on $L\left(\lambda_{i}\right)$.

Alternative proofs for exceptional groups. Here are very short proofs of Proposition 8.1 and Corollary 8.3 in case $G$ belongs to Table 1. By (5.4), $\tau$ is not zero on the irreducible representation $V$, so it is nondegenerate on $V$ and hence on all of $A(\mathfrak{g})$. Suppose $G$ is a compact real form; then every nonzero $G$-invariant bilinear form on the irreducible representation $V$ is definite, as can be seen by averaging. In particular, $\tau$ is definite on $V$ and so positive definite on $V$ by (5.4). Corollary 8.3 follows.

One says that $G$ is isotropic if it contains a copy of the one-dimensional split torus $\mathbb{G}_{\mathrm{m}}$ defined over $k$, and anisotropic otherwise. In case $k=\mathbb{R}, G$ is anisotropic if and only if it is compact. The following example provides something like a converse to Corollary 8.3. 
Example 8.4. Suppose $G$ is not of type $A_{1}$ and $G$ is isotropic; we claim that $\tau$ is isotropic. As $G$ is not of type $A_{1}, A(\mathfrak{g}) / k$ is not the trivial representation of $G$ as in the proof of Proposition 7.2, so $G$ acts on it with finite kernel. It follows that there is a nonzero subspace $U$ of $A(\mathfrak{g})$ on which $\mathbb{G}_{\mathrm{m}}$ acts with only positive weights or only negative weights, implying that $\tau\left(u, u^{\prime}\right)=0$ for $u, u^{\prime} \in U$ : that is, $\tau$ is isotropic.

The next example shows that the case $k=\mathbb{R}$ in Corollary 8.3 is somewhat special.

Example 8.5. We will show that $\tau$ may be isotropic, even if the group $G$ is anisotropic. Specifically, let $k$ be a number field and pick an odd number $n \geq 3$. There is an associative division algebra $D$ with center $k$ such that $\operatorname{dim}_{k} D=n^{2}$. The group $G=\mathrm{SL}_{1}(D)$ of norm 1 elements of $D$ is simply connected of type $A_{n-1}$ and is anisotropic. However, the group is split at every real place, so $\tau$ is isotropic at every real place (Example 8.4 ). As $\operatorname{dim} A(\mathfrak{g}) \geq 1+\operatorname{dim} \mathfrak{g}>5$, the form $\tau$ is isotropic over $k$ by the Hasse-Minkowski theorem.

We conclude the section with another corollary of Proposition 8.1.

Corollary 8.6. $A(\mathfrak{g})$ is a simple $k$-algebra.

Proof. The nondegeneracy of $\tau$ and Proposition 7.2 verify the hypotheses of Proposition A.10.

\section{The group scheme $\operatorname{Aut}(A(\mathfrak{g}))$}

There is a natural homomorphism $G \rightarrow \operatorname{Aut}(A(\mathfrak{g}))$. It has a finite kernel, the center of $G$, and it is injective if and only if $G$ is adjoint. The point of the following result is that in some cases, this homomorphism is an isomorphism.

Proposition 9.1. If $G$ has type $F_{4}$ or $E_{8}$, then $\operatorname{Aut}(A(\mathfrak{g}))=G$.

It follows trivially that for $G, G^{\prime}$ of type $F_{4}$ or $E_{8}$, we have: $G \cong G^{\prime}$ if and only if $A(\mathfrak{g}) \cong A\left(\mathfrak{g}^{\prime}\right)$.

Proof of Proposition 9.1. The number $\operatorname{dim} A(\mathfrak{g})$ is not zero in $k$, so as in Example A.6 $\operatorname{Aut}(A(\mathfrak{g}))$ is the sub-group-scheme of GL $(V)$ preserving the commutative product · on $V$ (nonzero by Proposition 5.3(1)) as well as the $G$-invariant bilinear form. In case $G$ has type $F_{4}$ or $E_{8}$, it is known that $G$ is the automorphism group of this product by [19, Lemma 5.1, Remark 5.5, and Section 7].

Here is what happens when the argument in the preceding proof is applied to $G$ of the other types in Table 1: for $G$ adjoint of type $E_{6}$, the argument shows that $G$ is the identity component of $\operatorname{Aut}(A(\mathfrak{g}))$. For $G$ of type $G_{2}$ or $E_{7}$, there is a copy of $\mathrm{SO}_{7}$ or $\mathrm{Sp}_{56} / \mu_{2}$ in $\mathrm{GL}(V)$ containing $G$ and preserving a nontrivial linear map $V \otimes V \rightarrow V$; as $G$ preserves a two-dimensional space of such products, the argument provided here is inconclusive in these cases.

For type $A_{2}, \operatorname{Aut}(A(\mathfrak{g}))$ is the orthogonal group $O(\mathfrak{g})$, whose identity component has type $D_{4}$; see Example 10.9.

Remark 9.2. Let $B$ be a simple, commutative and power-associative algebra over $\mathbb{C}$. Then by [2] and [27], $B$ is a Jordan algebra. The classification of such from [22, p. 204, Cor. 2] or [41, Section 13, 14] shows that the identity component of $\operatorname{Aut}(B)$ cannot be a simple group of type $G_{2}, E_{6}, E_{7}$ or $E_{8}$.

This provides an alternative argument that $A(\mathfrak{g})$ is not power-associative when $G$ is simple of type $E_{8}$ over $\mathbb{C}$, because $A(\mathfrak{g})$ is simple (Corollary 8.6) and commutative. (Compare Example 4.9.)

\section{Construction \#2: $A(\mathfrak{g})$ in $\operatorname{End}(V)$}

In this section, we leverage a common property of exceptional groups $G$ observed by Okubo to describe $A(\mathfrak{g})$ inside of $\operatorname{End}(V)$ for certain small $V$.

Suppose for this paragraph that $k=\mathbb{C}$ and $\pi: G \rightarrow \mathrm{GL}(V)$ is a representation. The maps $X \mapsto$ $\operatorname{Tr}\left(\pi(X)^{d}\right)$ are $G$-invariant homogeneous polynomial functions on $\mathfrak{g}$. It is standard that $k[\mathfrak{g}]^{G}$ is a polynomial ring with homogeneous generators. The smallest nonconstant generator can be taken to be $X \mapsto K(X, X)$ of degree 2 , and therefore an identity of the form $\operatorname{Tr}\left(\pi(X)^{2}\right)=c_{\pi} K(X, X)$ for all 
$X \in \mathfrak{g}$, where $c_{\pi}$ depends on $\pi$, as in Lemma 2.9(2) is inevitable. Similarly, for $G$ as in Table 1, the homogeneous generators of $k[\mathfrak{g}]^{G}$ are $X \mapsto K(X, X)$ of degree 2, for type $A_{2}$ one of degree 3, and no generators of degree 4 , and therefore there is an identity of the form $\operatorname{Tr}\left(\pi(X)^{4}\right)=\alpha_{\pi} K(X, X)^{2}$ for $X \in \mathfrak{g}$, where $\alpha_{\pi}$ depends only on $\pi$.

Okubo calculated the value of $\alpha_{\pi}$ in [34] in case $k=\mathbb{C}$; see also [33]. Here we note that the same result holds over our more general $k$. In this section, let $R$ denote the local $\operatorname{ring} \mathbb{Z}_{(\text {char } k \text { ) }}$ as in Lemma 2.8.

Lemma 10.1. Suppose $G$ is one of the types listed in Table 1 and that the representation $\pi: G \rightarrow \operatorname{GL}(V)$ is equivalent to $H^{0}(\lambda)$ or $V(\lambda)$ over the algebraic closure of $k$ for some dominant weight $\lambda$. Put $\mu_{\pi}:=\langle\lambda \mid \lambda+\delta\rangle$. If the rational number

$$
\alpha_{\pi}:=\frac{\left(6 \mu_{\pi}-1\right) \mu_{\pi} \operatorname{dim} V}{2(2+\operatorname{dim} G)(\operatorname{dim} G)}
$$

belongs to $R$, then $\operatorname{Tr}\left(\pi(X)^{4}\right)=\alpha_{\pi} K(X, X)^{2}$ for all $X \in \mathfrak{g}$. If additionally $G$ does not have type $A_{2}$, then

$$
\operatorname{Tr}\left(\pi(X)^{2} \pi(Y)^{2}\right)=-\frac{\mu_{\pi} \operatorname{dim} V}{6 \operatorname{dim} G} K([X, Y],[X, Y])+\frac{2 \alpha_{\pi}}{3} K(X, Y)^{2}+\frac{\alpha_{\pi}}{3} K(X, X) K(Y, Y)
$$

for $X, Y \in \mathfrak{g}$.

Sketch of proof. Similar to the proof of Lemma 2.9, let $G_{R}, \pi_{R}$ be lifts of $G, \pi$ to $R$, and put $K_{R}$ for the Killing form on $\mathfrak{g}_{R}$. The map $X \mapsto \operatorname{Tr}\left(\pi_{R}(X)^{4}\right)-\alpha_{\pi} K_{R}(X, X)$ is a polynomial function on $\mathfrak{g}_{R}$ (an element of $R\left[\mathfrak{g}_{R}\right]$ ) that vanishes over $\mathbb{C}$ by Okubo, so it is 0 in $R\left[\mathfrak{g}_{R}\right]$. Similarly, equation (10.2) holds over $\mathbb{C}$; see [33, p. 284], so it also holds over $R$.

Example 10.3. For the adjoint representation, we have

$$
\alpha_{\text {Ad }}=\frac{5}{2(2+\operatorname{dim} G)},
$$

which belongs to $R$ for $G$ as in Table 1 . (In case $G$ has type $A_{2}, \alpha_{\text {Ad }}=1 / 4$. For the other types, $\operatorname{dim} G+2$ is of the form $2^{x} 3^{y} 5^{z}$ for some $x, y, z$.) Rewriting a formula for $\operatorname{dim} G$ in terms of $h^{\vee}$ from [10, p. 431] or the polynomial in $[34,3.17]$ produces this remarkable formula:

$$
4 \alpha_{\text {Ad }}\left(h^{\vee}\right)^{2}=h^{\vee}+6 .
$$

(This is just one example from many families of formulas; compare, for example, [12], [13], [29] and [30].)

Here is the promised embedding.

Proposition 10.5. If $G$ has type $A_{2}, G_{2}, F_{4}, E_{6}$ or $E_{7}$ and $\pi: G \rightarrow \mathrm{GL}(V)$ is an irreducible representation of dimension 3, 7, 26, 27 or 56, respectively, then the formula

$$
\sigma(S(X Y))=6 h^{\vee} \pi(X) \bullet \pi(Y)-\frac{1}{2} K(X, Y) \operatorname{Id}_{B} \quad \text { for } X \in \mathfrak{g} .
$$

defines an injective G-equivariant linear map

$$
\sigma: A(\mathfrak{g}) \hookrightarrow \operatorname{End}(V) .
$$

If additionally $G$ is not of type $A_{2}$, then $\sigma$ satisfies

$$
\operatorname{proj}_{\pi(\mathfrak{g})}\left(\sigma\left(S\left(X^{2}\right)\right) \bullet \pi(Y)\right)=\pi\left(S\left(X^{2}\right) Y\right) \quad \text { for } Y \in \mathfrak{g} .
$$


Proof. The representation $\pi$ is irreducible. Moreover, one checks that in each case we have:

$$
\mu_{\pi}=\frac{h^{\vee}+1}{h^{\vee}+6}
$$

which by (10.4) is the same as

$$
h^{\vee}=\frac{2+d}{2\left(6 \mu_{\pi}-1\right)}=\frac{\mu_{\pi} d_{\pi}}{4 \alpha_{\pi} d}
$$

where we have abbreviated $d_{\pi}:=\operatorname{dim} V$ and $d:=\operatorname{dim} G$.

Recall that $\mathrm{S}^{2} \mathfrak{g}=k \oplus L(\lambda) \oplus L(2 \widetilde{\alpha})$ as a representation of $G$, whereas, at least in case $k=\mathbb{C}$, $\operatorname{End}(V)$ and $A(\mathfrak{g})$ contain $k$ and $L(\lambda)$ with multiplicity 1 and do not contain $L(2 \widetilde{\alpha})$. It follows that any $G$-equivariant linear map $S^{2} \mathfrak{g} \rightarrow \operatorname{End}(V)$ factors through $S: S^{2} \mathfrak{g} \rightarrow A(\mathfrak{g})$. In particular, the map $X Y \mapsto 6 h^{\vee} \pi(X) \bullet \pi(Y)-\frac{1}{2} K(X, Y) \operatorname{Id}_{V}$ does so, whence the map $\sigma$ from (10.6) is well defined. This $\sigma$ is defined over $R$, and so it is also well defined for $k$.

We now verify (10.7), so assume $G$ is not of type $A_{2}$. Linearizing (10.2) in $Y$ gives

$$
\begin{aligned}
\operatorname{Tr}\left(\left(\pi(X)^{2} \bullet \pi(Y)\right) \pi(Z)\right)= & -\frac{\mu_{\pi} d_{\pi}}{6 d} K([X, Y],[X, Z]) \\
& +\frac{2 \alpha_{\pi}}{3} K(X, Y) K(X, Z)+\frac{\alpha_{\pi}}{3} K(X, X) K(Y, Z) .
\end{aligned}
$$

As $K(Y, Z)=\frac{d}{\mu_{\pi} d_{\pi}} \operatorname{Tr}(\pi(Y) \pi(Z))($ Lemma 2.9), we have

$$
\frac{\alpha_{\pi}}{3} K(X, X) K(Y, Z)=\operatorname{Tr}\left(\left(\frac{d \alpha_{\pi}}{3 \mu_{\pi} d_{\pi}} K(X, X) \operatorname{Id}_{B} \bullet \pi(Y)\right) \pi(Z)\right) .
$$

We obtain

$$
\operatorname{Tr}\left(\left(\left(\pi(X)^{2}-\frac{d \alpha_{\pi}}{3 \mu_{\pi} d_{\pi}} K(X, X) \operatorname{Id}_{B}\right) \bullet \pi(Y)\right) \pi(Z)\right)=\frac{2 \alpha_{\pi}}{3} K\left(\left(\frac{\mu_{\pi} d_{\pi}}{4 \alpha_{\pi} d}(\operatorname{ad} X)^{2}+P\left(X^{2}\right)\right) Y, Z\right) .
$$

Multiplying both sides by $6 h^{\vee}$ and applying (10.8) gives (10.7).

Example 10.9 $\left(A\left(\mathfrak{s l}_{3}\right)\right)$. The case $\mathfrak{g}=\mathfrak{s l}_{3}$ was included in Table 1 but excluded from Section 5, so we now use the preceding construction to describe $A\left(\mathfrak{s l}_{3}\right)$. For $X, Y \in \mathfrak{s l}_{3}, \operatorname{Tr}(X Y)=\frac{1}{6} K(X, Y)$ by Lemma 2.9, so the embedding $\sigma: A\left(\mathfrak{s l}_{3}\right) \rightarrow M_{3}(k)$ is via

$$
\sigma\left(S\left(X^{2}\right)\right)=18 X^{2}-3 \operatorname{Tr}\left(X^{2}\right) I
$$

and it is an isomorphism by dimension count. We define a product $*$ on $M_{3}(k)$ via $P * Q:=\sigma^{-1}(P) \diamond$ $\sigma^{-1}(Q)$. Putting $\varepsilon:=\frac{1}{3} \operatorname{Tr}$ for the counit and chasing through the formulas, we find:

$$
P * Q=\left[\frac{1}{2} \varepsilon(P \bullet Q)-\frac{3}{2} \varepsilon(P) \varepsilon(Q)\right] I+\varepsilon(Q) P+\varepsilon(P) Q .
$$

That is, $M_{3}(k)$ with the multiplication $*$ is of the form $\mathcal{U}\left(\mathfrak{s l}_{3}, f\right)$ with notation as in the appendix, where the multiplication on $\mathfrak{s l}_{3}$ is taken to be identically zero and $f(P, Q)=\frac{1}{2} \varepsilon(P \bullet Q)$. This is the Jordan algebra constructed from the bilinear form $f$ as in [22, pp. 13, 14] see also Remark A.11.

\section{Final remarks}

We have defined here a construction that takes a simple algebraic group $G$ (equivalently, a simple Lie algebra $\mathfrak{g}$ ) over a field $k$, with mild hypotheses on the field $k$, and gives an explicit formula (4.1) for the multiplication on a unital $k$-algebra $A(\mathfrak{g})$ on which $G$ acts by automorphisms. We used the description 
of $A(\mathfrak{g})$ as a representation of $G$ to show that it is a simple algebra, that the bilinear form on it is nondegenerate, and that for $G$ of type $F_{4}$ or $E_{8}$ the automorphism group is exactly $G$.

\section{Computation}

One can construct $A(\mathfrak{g})$ using a computer in a way amenable to computations as follows. First, construct $G$ or $\mathfrak{g}$ together with its adjoint representation or, in the cases where Proposition 10.5 applies, its natural representation. Pick a basis $\left\{X_{i}\right\}$ of $\mathfrak{g}$, and compute $S\left(X_{i} X_{j}\right) \in \operatorname{End}(\mathfrak{g})$ in the first case or $\sigma\left(S\left(X_{i} X_{j}\right)\right) \in \operatorname{End}(B)$ in the second, for $i \leq j$. Among these elements, select a maximal linearly independent subset; it is a basis for $A(\mathfrak{g})$. For each pair of basis elements, one may calculate the product $\diamond$ using (4.1) and express the result in terms of the chosen basis. This gives the 'structure constants' for the algebra. Magma [5] code implementing this recipe can be found at github.com/skipgaribaldi/chayet-garibaldi.

\section{Polynomial identities}

Among the algebras $A(\mathfrak{g})$ for $G$ in Deligne's exceptional series, the cases $A\left(\mathfrak{s l}_{2}\right)$ and $A\left(\mathfrak{s l}_{3}\right)$ are unusual for being Jordan algebras and in particular power-associative, whereas $A(\mathfrak{g})$ is not power-associative for other choices of $\mathfrak{g}$ (Proposition 5.3(2)). It is natural, then, to ask what identities $A(\mathfrak{g})$ does satisfy in those cases. It does not satisfy any polynomial identity of degree $\leq 4$ that is not implied by commutativity (Proposition A.8). Moreover, in the case $G=G_{2}$, we verified using a computer that $A(\mathfrak{g})$ and also $\mathcal{U}(V, c f)$ for every $c \neq 1$ do not satisfy any degree 5 identity not implied by commutativity, leveraging the classification of such identities from [36, Th. 5].

In case $G=G_{2}$ or $E_{8}$, the $G$-module $S^{2} V$ has only six summands, which suggests the existence of an identity of degree $\leq 7$ in view of Example A.9. In the case of $G_{2}$, the 26 nonassociative and commutative monomials of degree $\leq 7$ in an element $a \in A\left(\mathfrak{g}_{2}\right)$ are linearly dependent. We have found a 'weighted' polynomial identity for $A\left(\mathfrak{g}_{2}\right)$ in the sense of [47]: that is, for each nonassociative monomial $m$ of degree $\leq 7$, there is a polynomial function $\phi_{m}$ on $A\left(\mathfrak{g}_{2}\right)$ so that the function $a \mapsto \sum_{m} \phi_{m}(a) m(a)$ is identically zero. It would be interesting to know whether a similar identity holds for $A\left(\mathfrak{e}_{8}\right)$.

\section{Appendix A. Adjoining a unit to a $k$-algebra}

We carefully record in this appendix some details concerning adjoining a multiplicative identity to a $k$-algebra, because we do not know a sufficient reference for this material. Suppose we are given a $k$ algebra $V$ that may not contain a multiplicative identity. That is, $V$ is a vector space over $k$ together with a $k$-bilinear map $: V \times V \rightarrow V$, which we call the multiplication on $V$. Given a bilinear form $f$ on $V$, we define a unital $k$-algebra $\mathcal{U}(V, f)$ that has underlying vector space $k \oplus V$ and multiplication

$$
\left(x_{0}, x_{1}\right)\left(y_{0}, y_{1}\right)=\left(x_{0} y_{0}+f\left(x_{1}, y_{1}\right), x_{0} y_{1}+y_{0} x_{1}+x_{1} \cdot y_{1}\right)
$$

for $x_{0}, y_{0} \in k$ and $x_{1}, y_{1} \in V$. Then $(1,0)$ is the multiplicative identity in $\mathcal{U}(V, f)$ and $V$ is a subalgebra.

Remark A.2. The construction $\mathcal{U}(V, f)$ is discussed from a different point of view in Fox's paper [17, Section 5]. A specific example of this construction in earlier literature comes from the 196883dimensional Griess algebra $V$, whose automorphism group is the Monster. Fox points out (Example 5.7) that various choices of $f$ are used in the literature when authors add a unit to $V$.

In the literature, one commonly finds the more restrictive recipe $\mathcal{U}(V, 0)$ for adjoining a unit to $V$ (that is, where $f$ is identically zero); see, for example, [39, Ch. II]. This has the advantage of not introducing the parameter $f$; however, it has the disadvantage of always producing a non-simple algebra $-V$ is an ideal in $\mathcal{U}(V, 0)$ - and therefore it does not produce popular examples of simple algebras like the $n$-by- $n$ matrices over a field, the octonions or Albert algebras. For more on this, see Proposition A.10 below. 
Remark A.3. One could imagine generalizing the construction to add a further parameter $\mu \in k$ and defining $\mathcal{U}(V, f, \mu)$ to have the same underlying vector space as $\mathcal{U}(V, f)$ but with multiplication rule

$$
\left(x_{0}, x_{1}\right)\left(y_{0}, y_{1}\right)=\left(x_{0} y_{0}+f\left(x_{1}, y_{1}\right), x_{0} y_{1}+y_{0} x_{1}+\mu x_{1} y_{1}\right) \text {. }
$$

It is easily seen, however, that $\mathcal{U}(V, f, \mu)$ is isomorphic to $\mathcal{U}\left(V, \mu^{-2} f\right)$, so no generality would be gained.

Throughout the remainder of this section, we assume that all algebras considered are finitedimensional.

\section{Counit}

For a $k$-algebra $A$ with multiplicative identity $e$, we call a $k$-linear map $\varepsilon: A \rightarrow k$ such that $\varepsilon(e)=1$ a counit. Such a map gives a direct sum decomposition $A=k e \oplus V$ as vector spaces where $V:=\operatorname{ker} \varepsilon$ and furthermore expresses $A$ as an algebra $U(V, f)$ by setting

$$
f\left(v, v^{\prime}\right):=\varepsilon\left(v v^{\prime}\right) \quad \text { and } \quad v \cdot v^{\prime}:=v v^{\prime}-f\left(v, v^{\prime}\right) \text { for } v, v^{\prime} \in V .
$$

Conversely, every algebra $U(V, f)$ has a natural counit, namely the projection of $k \oplus V$ on its first factor. In this way, we may identify the notions of unital $k$-algebras with a counit on the one hand and algebras of the form $U(V, f)$ (with specified $V$ and $f$ ) on the other.

Additionally, a counit defines a bilinear form $\tau$ on $A$ by setting

$$
\tau\left(a, a^{\prime}\right):=\varepsilon\left(a a^{\prime}\right) \quad \text { for all } a, a^{\prime} \in A .
$$

Evidently, the direct sum decomposition $A=k e \oplus V$ is an orthogonal sum with respect to $\tau$ : that is, $\tau(e, v)=0$ for all $v \in V$, and the restriction of $\tau$ to $V$ is $f$. From this it follows that $\tau$ is symmetric (respectively, nondegenerate) if and only if $f$ is symmetric (respectively, nondegenerate).

Example A.6. In the special case where the integer $\operatorname{dim} A$ is not zero in $k$, there is a natural counit $\varepsilon: a \mapsto \frac{1}{\operatorname{dim} A} \operatorname{Tr}\left(M_{a}\right)$, where we have written $M_{a} \in \operatorname{End}(A)$ for the linear transformation $b \mapsto a b$ Therefore there is a natural way of writing $A$ as $\mathcal{U}(V, f)$ for $V$ and $f$ as in (A.4). Moreover, every algebra automorphism of $A$ preserves $\varepsilon$, whence the group scheme $\operatorname{Aut}(A)$ is identified with the sub-groupscheme of GL( $V)$ of transformations that preserve both the multiplication - and the bilinear form $f$.

Recall that a bilinear form on a $k$-algebra is called associative if it satisfies (6.2).

Proposition A.7. In the notation of the preceding four paragraphs, $\tau$ is associative (with respect to the algebra $A$ ) if and only if $f$ is associative (with respect to the algebra $V$ ).

Proof. Write elements $a, a^{\prime}, a^{\prime \prime} \in A$ as $a=\left(a_{0}, a_{1}\right)$, etc. Then $\tau\left(a a^{\prime}, a^{\prime \prime}\right)-\tau\left(a, a^{\prime} a^{\prime \prime}\right)=f\left(a_{1}\right.$. $\left.a_{1}^{\prime}, a_{1}^{\prime \prime}\right)-f\left(a_{1}, a_{1}^{\prime} \cdot a_{1}^{\prime \prime}\right)$.

The property of being metrized - that is, of having a nondegenerate and associative bilinear form — has the following interesting consequence.

Proposition A.8. Let $A$ be a commutative $k$-algebra where char $k \neq 2,3,5$, and suppose that $A$ is metrized. If $A$ satisfies an identity of degree $\leq 4$ not implied by commutativity, then $A$ satisfies the Jordan identity $x\left(x^{2} y\right)=x^{2}(x y)$ and is power-associative.

Proof. Writing $\top$ for the involution on $\operatorname{End}(A)$ corresponding to the nondegenerate associative bilinear form on $A$, we have $M_{a}^{\top}=M_{a}$ and $\left(M_{a} M_{b}\right)^{\top}=M_{b} M_{a}$ for all $a, b \in A$. Note that the Jordan identity is equivalent to the assertion that $\left[M_{a}, M_{a^{2}}\right]=0$ for all $a \in A$.

According to [36, Th. 4], $A$ satisfies (A.12) or

7. $2((y x) x) x+y x^{3}=3\left(y x^{2}\right) x$ or 
8. $2\left(y^{2} x\right) x-2((y x) y) x-2((y x) x) y+2\left(x^{2} y\right) y-y^{2} x^{2}+(y x)^{2}=0$.

Identity 7 is equivalent to the statement $2 M_{x}^{3}+M_{x^{3}}=3 M_{x} M_{x^{2}}$. Applying $\top$ to this identity, subtracting it, and dividing by 3 , we obtain $\left[M_{x}, M_{x^{2}}\right]=0$.

For (A.12), replacing $a$ with $x+y$, expanding, and taking the terms of degree 1 in $y$, we find $M_{x^{3}}+M_{x} M_{x^{2}}+2 M_{x}^{3}=4 M_{x^{2}} M_{x}$. Applying $T$ to this identity, subtracting it, and dividing by 5 gives $\left[M_{x}, M_{x^{2}}\right]=0$.

Finally, if Identity 8 holds, then replacing $y$ with $y+z$ and taking the terms of degree 1 in $y$ and $z$, replacing $y$ with $x$, and applying the same procedure as in previous cases again gives $\left[M_{x}, M_{x^{2}}\right]=0$.

For comparison, the situation when $A$ is not assumed to be metrized is more complicated; see [37] and [8].

The following example provides a positive statement.

Example A.9. Let $A$ be a commutative $k$-algebra that is metrized, and suppose that the $\operatorname{Aut}(A)$-module $\mathrm{S}^{2} A$ has a composition series of length $d$. Define $P_{e}: \mathrm{S}^{e} A \rightarrow \operatorname{End}(A)$ via

$$
P\left(a_{1} a_{2} \cdots a_{e}\right):=\sum_{\text {permutations } \sigma} M_{a_{\sigma(1)}} M_{a_{\sigma(2)}} \cdots M_{a_{\sigma(e)}}
$$

This is $\operatorname{Aut}(A)$-equivariant, and its image $H_{e}$ is contained in the space of symmetric operators on $A$ with respect to $\tau$, which we identify with $S^{2} A$. Setting $H_{0}:=k \operatorname{Id}_{A}$ and $I_{e}:=H_{0}+H_{1}+\cdots+H_{e}$, we obtain an increasing chain of submodules $0 \neq I_{0} \subsetneq I_{1} \subseteq \cdots$ so that $I_{e}=I_{e+1}$ for some $e<d$. That is, a symmetric expression

$$
\sum_{\sigma} a_{\sigma(1)}\left(a_{\sigma(2)}\left(a_{\sigma(3)} \cdots\left(a_{\sigma(e+1)} b\right)\right) \cdots\right) \quad \in A,
$$

where each summand is a product of at most $d+1$ terms, can be expressed in terms of symmetric expressions in the $a$ s involving products of fewer terms.

\section{Simplicity}

A $k$-algebra $A$ is simple if the only two-sided ideals in $A$ are 0 and $A$ itself. We prove the following criterion for simplicity.

Proposition A.10. Let A be a unital k-algebra with counit $\varepsilon$. If

1. There is a connected group scheme $G \subseteq \operatorname{Aut}(A)$ that stabilizes $\varepsilon$;

2. $k$ is not a composition factor of $\operatorname{ker} \varepsilon$ as a $G$-module; and

3. $\tau$ as defined in (A.5) is nondegenerate,

then $A$ is simple.

Remark A.11. In the case where the multiplication on $V:=\operatorname{ker} \varepsilon$ is identically zero, the algebra $A$ is of the kind studied in [24].

Proof of Proposition A.10. Put $V:=\operatorname{ker} \varepsilon$. We first claim that every $G$-invariant subspace $I$ of $A$ is a direct $\operatorname{sum} I=(k e \cap I) \oplus(V \cap I)$. If the restriction of the projection $1-\varepsilon: A \rightarrow V$ to $I$ has a kernel, then $\operatorname{ker}(1-\varepsilon)=k e$ is contained in $I$ and the claim is clear. Otherwise, $1-\varepsilon$ is injective and $I=\{(\pi(w), w)\}$ for $w \in W:=(1-\varepsilon)(I)$ and some $G$-equivariant linear map $\pi: W \rightarrow k$. By (2), however, $\pi$ must be zero, and the claim follows.

We next verify that every nonzero and $G$-invariant ideal $I$ of $A$ is equal to $A$. By the preceding paragraph, we may suppose that there is a nonzero $v \in V \cap I$. Since $\tau$ is nondegenerate, there is an $a \in A$ so that $0 \neq \tau(v, a)=\varepsilon(v a)$. That is, $v a$ is a nonzero element of $k e \cap I$, whence $I=A$. 
Now let $I$ be a nonzero ideal in $A$. The sum of $G$-conjugates of $I, \sum_{g} g I$ is a nonzero and $G$-invariant ideal, so it equals $A$. We conclude that $I$ itself equals $A$ by arguing as in the proof of [38, Theorem 5], which concerns the analogous case of a non-unital algebra that is an irreducible representation of a connected group.

\section{Power-associativity}

A $k$-algebra $A$ is power-associative if the subalgebra generated by any element $a \in A$ is associative. It is strictly power-associative if $A \otimes_{k} F$ is power-associative for every field $F$ containing $k$. We now focus on the case where $A$ is commutative, as is the algebra $A(\mathfrak{g})$ elsewhere in this paper, and as is the algebra $\mathcal{U}(V, f)$ when $V$ is commutative and $f$ is symmetric.

If $A$ is power-associative, then in particular

$$
a(a(a a))-(a a)(a a)=0 \quad \text { for all } a \in A .
$$

When char $k \neq 2,3,5$, (A.12) is equivalent to $A$ being strictly power-associative [1, Th. 1], see also [26, p. 364].

The property of whether $\mathcal{U}(V, f)$ is strictly power-associative is rather constrained. In the proposition below, we write $v^{2}$ for the element $v \cdot v \in V$.

Proposition A.13. Suppose $f$ is not alternating. If the polynomial map $v \mapsto v \wedge v^{2} \in \wedge^{2} V$ is not identically zero, then there is at most one $c \in k$ so that $U(V, c f)$ is strictly power-associative.

Proof. We focus on (A.12) for $a \in \mathcal{U}(V, c f)$. Writing out $a=\left(a_{0}, a_{1}\right)$ and expanding $a(a(a a))-$ $(a a)(a a)$, we find $\left(c\left(f\left(a_{1}, a_{1}\left(a_{1}^{2}\right)\right)-f\left(a_{1}^{2}, a_{1}^{2}\right)\right), x+c y\right)$ for

$$
x=a_{1}\left(a_{1} a_{1}^{2}\right)-a_{1}^{2} a_{1}^{2} \quad \text { and } \quad y=f\left(a_{1}, a_{1}^{2}\right) a_{1}-f\left(a_{1}, a_{1}\right) a_{1}^{2} .
$$

By hypothesis, $a_{1}, a_{1}^{2}$ are linearly independent for generic $a_{1} \in V$. And $f\left(a_{1}, a_{1}\right)$ is also nonzero for generic $a_{1} \in V$ because $f$ is not alternating, so we conclude that $y$ is not the zero polynomial on $V$. It follows that the polynomial function $x+c y$ on $V$ is identically zero for at most one value of $c \in k$.

Remark A.15. For $a=\left(a_{0}, a_{1}\right) \in \mathcal{U}(V, f)$, we have $(1,0) \wedge a \wedge a^{2}=(1,0) \wedge\left(0, a_{1}\right) \wedge\left(0, a_{1}^{2}\right)$ in $\wedge^{3} \mathcal{U}(V, f)$, where the squaring operation on the left side is relative to the multiplication on $\mathcal{U}(V, f)$ and on the right side is relative to the multiplication - on $V$. As a consequence, the hypothesis of Proposition A.13 can be phrased in the equivalent form: the polynomial map $a \mapsto(1,0) \wedge a \wedge a^{2}$ is not zero.

Remark A.16. If the squaring map $v \mapsto v^{2}$ is the zero function, the identities $a^{2} a=a a^{2}$ and (A.12) hold in $\mathcal{U}(V, c f)$. If char $k=0$, it follows that $\mathcal{U}(V, c f)$ is strictly power-associative for every $c \in k$ by [1, Th. 2].

The following lemma allows one to apply Proposition A.13 in situations such as that in Proposition A.10, by taking $F(v)=v^{2}$.

Lemma A.17. Suppose $\operatorname{dim} V \geq 2$, and let $F$ be a $G$-equivariant polynomial function $V \rightarrow V$ that is homogeneous of degree $d \geq 1$. If the polynomial map $v \mapsto v \wedge F(v)$ is identically zero, then there is a $G$-invariant polynomial function $\bar{F}: V \rightarrow k$ that is homogeneous of degree $d-1$ and $F(v)=\bar{F}(v) v$ for all $v \in V \otimes K$ for every extension $K$ of $k$.

Proof. There is a $G$-invariant function $\bar{F}: V \backslash\{0\} \rightarrow k$ defined implicitly by the equation $\bar{F}(v) v=F(v)$. We argue that it is a polynomial function on $V$.

Fix a basis $x_{1}, \ldots, x_{n}$ of $V^{*}$. The $i$ th coordinate $\left.x_{i}\right|_{F(v)}$ of $F(v)$ is $f_{i}(v)$ for some homogeneous degree $d$ polynomial $f_{i} \in k\left[x_{1}, \ldots, x_{n}\right]$. On the open set $U_{i}$, where $x_{i}$ does not vanish, $\bar{F}=f_{i} / x_{i}$. For 
$i \neq j, f_{i} / x_{i}$ and $f_{j} / x_{j}$ agree on $U_{i} \cap U_{j}$, so $x_{i} f_{j}=x_{j} f_{i}$ in the polynomial ring. As $x_{i}$ does not divide $x_{j}$, it must divide $f_{i}$. Setting $\bar{f}_{i}:=f_{i} / x_{i}$, the polynomial function $v \mapsto F(v)-\bar{f}_{i}(v) v$ is zero on $U_{i}$, so it is zero on $V$ : that is, $\bar{F}: V \rightarrow k$ is a polynomial.

Acknowledgements. We thank Robert Guralnick and Benedict Gross for helpful conversations, which improved some of the proofs, and anonymous referees for their comments, which improved the exposition.

Conflict of Interest: None.

Financial support: None.

\section{References}

[1] A.A. Albert, On the power-associativity of rings, Summa Brasil. Math. 2 (1948), no. 2, 21-32.

[2] A.A. Albert, A theory of power-associative commutative algebras, Trans. Amer. Math. Soc. 69 (1950), $503-527$.

[3] J.C. Baez and J. Huerta, $\mathrm{G}_{2}$ and the rolling ball, Trans. Amer. Math. Soc. 366 (2014), no. 10, 5257-5293.

[4] A. Borel and J. Tits, Groupes réductifs, Publ. Math. IHES 27 (1965), 55-150.

[5] W. Bosma, J. Cannon and C. Playoust, The Magma algebra system I: the user language, J. Symbolic Computation 9 (1990), 677-698.

[6] N. Bourbaki, Lie groups and Lie algebras: Chapters 4-6, Springer-Verlag, Berlin, 2002.

[7] N. Bourbaki, Lie groups and Lie algebras: Chapters 7-9, Springer-Verlag, Berlin, 2005.

[8] L. Carini, I.R. Hentzel and G.M. Piacentini Cattaneo, Degree four identities not implied by commutativity, Comm. Algebra 16 (1988), no. 2, 339-356.

[9] E. Cartan, Les groupes réels simples finis et continus, Ann. Sci. École Norm. Sup. (3) 31 (1914), 265-355.

[10] A.M. Cohen and R. de Man, Computational evidence for Deligne's conjecture regarding exceptional Lie groups, C. R. Acad. Sci. Paris Sér. I Math. 322 (1996), no. 5, 427-432.

[11] T. De Medts and M. Van Couwenberghe, Non-associative Frobenius algebras for simply laced Chevalley groups, arxiv:2005.02625, May 2020.

[12] P. Deligne, La série exceptionnelle de groupes de Lie, C. R. Acad. Sci. Paris Sér. I Math. 322 (1996), no. 4, $321-326$.

[13] P. Deligne and B.H. Gross, On the exceptional series, and its descendants, C. R. Math. Acad. Sci. Paris 335 (2002), no. 11, 877-881.

[14] M. Demazure and A. Grothendieck, Schémas en groupes III: Structure des schemas en groupes reductifs, Société Mathématique de France, 2011, re-edition edited by P. Gille and P. Polo.

[15] E.B. Dynkin, Semisimple subalgebras of semisimple Lie algebras, Amer. Math. Soc. Transl. (2) 6 (1957), 111-244, [Russian original: Mat. Sbornik N.S. 30(72) (1952), 349-462].

[16] F. Engel, Ein neues, dem linearen Komplexe analoges Gebilde, Berichte über die Verhandlungen der Königlich Sächsischen Gesellschaft der Wissenschaften zu Leipzig. Mathematisch-Physische Klasse 52 (1900), 63- 76, 220 -239.

[17] D.J.F. Fox, Commutative algebras with nondegenerate invariant trace form and trace-free multiplication endomorphisms, arxiv:2004.12343, April 2020.

[18] S. Garibaldi, E8, the most exceptional group, Bull. Amer. Math. Soc. (N.S.) 53 (2016), no. 4, 643-671.

[19] S. Garibaldi and R.M. Guralnick, Simple groups stabilizing polynomials, Forum of Mathematics: Pi 3 (2015), e3 (41 pages).

[20] C. Herz, Alternating 3-forms and exceptional simple Lie groups of type $G_{2}$, Canad. J. Math. 35 (1983), no. 5, 776-806.

[21] G. Hiss, Die adjungierten Darstellungen der Chevalley-Gruppen, Arch. Math. (Basel) 42 (1984), 408-416.

[22] N. Jacobson, Structure and Representations of Jordan Algebras, Coll. Pub., vol. 39, Amer. Math. Soc., Providence, RI, 1968.

[23] J.C. Jantzen, Representations of Algebraic Groups, second edn, Math. Surveys and Monographs, vol. 107, Amer. Math. Soc., 2003.

[24] W.E. Jenner, On non-associative algebras associated with bilinear forms, Pacific J. Math. 10 (1960), no. 2, 573-575.

[25] W. Killing, Die Zusammensetzung der stetigen endlichen Transformationsgruppen. Zweiter Theil, Math. Ann. 33 (1889), $1-48$.

[26] L.A. Kokoris, New results on power-associative algebras, Trans. Amer. Math. Soc. 77 (1954), 363-373.

[27] L.A. Kokoris, Simple power-associative algebras of degree two, Ann. of Math. (2) 64 (1956), 544-550.

[28] Y. Krasnov and V.G. Tkachev, Variety of idempotents in nonassociative algebras, Topics in Clifford Analysis: Special Volume in Honor of Wolfgang Sprößig (S. Bernstein, ed.), Springer International Publishing, 2019, pp. 405-436.

[29] J.M. Landsberg and L. Manivel, Triality, exceptional Lie algebras, and Deligne dimension formulas, Adv. Math. 171 (2002), 59-85.

[30] J.M. Landsberg and L. Manivel, A universal dimension formula for complex simple Lie algebras, Adv. Math. 201 (2006), no. 2, 379-407.

[31] F. Lübeck, Small degree representations of finite Chevalley groups in defining characteristic, LMS J. Comput. Math. 4 (2001), 135-169.

[32] G.J. McNinch, Dimensional criteria for semisimplicity of representations, Proc. London Math. Soc. (3) 76 (1998), $95-149$. 
[33] K. Meyberg, Okubo's quartic trace formula for exceptional Lie algebras, J. Algebra 84 (1983), $279-284$.

[34] S. Okubo, Quartic trace identity for exceptional Lie algebras, J. Math. Phys. 20 (1979), 586-593.

[35] P. Orlik and L. Solomon, Singularities II: automorphisms of forms, Math. Ann. 231 (1978), 229-240.

[36] J.M. Osborn, Identities of non-associative algebras, Canad. J. Math. 17 (1965), 78-92.

[37] J.M. Osborn, Commutative non-associative algebras and identities of degree four, Canadian J. Math. 20 (1968), 769-794.

[38] V.L. Popov, An Analogue of M. Artin's Conjecture on Invariants for Nonassociative Algebras, Lie groups and Lie algebras: E. B. Dynkin's Seminar, Amer. Math. Soc. Transl. Ser. 2, vol. 169, Amer. Math. Soc., Providence, RI, 1995, pp. $121-143$.

[39] R. Schafer, An Introduction to Nonassociative Algebras, Dover, 1994.

[40] J-P. Serre, Galois Cohomology, Springer-Verlag, 2002, originally published as Cohomologie Galoisienne (1965).

[41] T.A. Springer, Jordan Algebras and Algebraic Groups, Ergebnisse der Mathematik und ihrer Grenzgebiete, vol. 75, SpringerVerlag, 1973.

[42] T.A. Springer, Linear Algebraic Groups, second edn, Birkhäuser, 1998.

[43] T.A. Springer and R. Steinberg, Conjugacy Classes, Seminar on Algebraic Groups and Related Finite Groups (The Institute for Advanced Study, Princeton, N.J., 1968/69), Lecture Notes in Math., vol. 131, Springer, Berlin, 1970, [= Robert Steinberg: collected papers, pp. 293-394], pp. 167-266.

[44] R. Steinberg, Lectures on Chevalley Groups, University Lecture Series, no. 66, American Math. Soc., Providence, RI, 2016.

[45] R. Suter, Coxeter and dual Coxeter numbers, Comm. Algebra 26 (1998), no. 1, 147-153.

[46] J. Tits, Représentations linéaires irréductibles d'un groupe réductif sur un corps quelconque, J. Reine Angew. Math. 247 (1971), 196-220.

[47] V.G. Tkachev, The universality of one half in commutative nonassociative algebras with identities, J. Algebra $\mathbf{5 6 9}$ (2021), 466-510.

[48] P. Vogel, The universal Lie algebra, unpublished, June 1999.

[49] P.W. Winter, On the modular representation theory of the two-dimensional special linear group over an algebraically closed field, J. London Math. Soc. (2) 16 (1977), no. 2, 237-252. 